\title{
Hemorragia subaracnoidea aneurismática: guía de tratamiento del Grupo de Patología Vascular
}

\section{de la Sociedad Española de Neurocirugía}

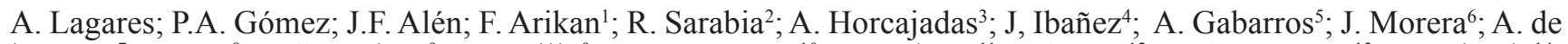 \\ la Lama'; L. Ley ${ }^{8}$; J. Gonçalves $;$; A. Maillo; ; J. Domínguez ${ }^{10}$; J.L. Llacer ${ }^{11}$; I. Arrese ${ }^{12}$; D. Santamarta ${ }^{13}$; P. Delgado ${ }^{14}$; \\ G. Rodríguez Boto ${ }^{15}$ y J. Vilalta
}

Hospital 12 de Octubre Madrid. Hospital Vall d'Hebron Barcelona ${ }^{1}$. Hospital Universitario Rio Hortega Valladolid². Hospital Virgen de las Nieves Granada ${ }^{3}$. Hospital Son Dureta Palma de Mallorca ${ }^{4}$. Hospital Bellvitge Barcelona ${ }^{5}$. Hospital Doctor Negrín Las Palmas de Gran Canaria ${ }^{6}$. Hospital Xeral Vigo $^{7}$. Hospital Puerta de Hierro Madrid ${ }^{8}$. Hospital Clínico Universitario Salamanca . Hospital Virgen de la Candelaria Tenerife ${ }^{10}$. Hospital Ribera Alcira ${ }^{11}$. Hospital Donosti San Sebastián ${ }^{12}$. Hospital Virgen Blanca León ${ }^{13}$. Hospital General Yagüe Burgos ${ }^{14}$. Hospital Clínico Universitario de Madrid ${ }^{15}$.

\section{Resumen}

Se realiza una actualización sobre los aspectos más importantes de la hemorragia subaracnoidea aneurismática respecto a las guías previamente publicadas por el grupo de trabajo de la SENEC. Las recomendaciones propuestas deben considerarse como una guía general de manejo de esta patología. Sin embargo, pueden ser modificadas, incluso de manera significativa por las circunstancias propias de cada caso clínico, o las variaciones en los recursos diagnósticos y terapéuticos del centro hospitalario que reciba al paciente.

PALABRAS CLAVE. Hemorragia subaracnoidea. Isquemia. Vasoespasmo. Aneurisma. Clip. Coil. Cirugía. SENEC.

Aneurysmal subarachnoid hemorrhage: group of study of Cerebrovascular Pathology of the Spanish Society of Neurosurgery management guideline

\section{Summary}

An actualized revision of the most important aspects of aneurismal subarachnoid hemorrhage is presented from the guidelines previously published by the group of study of cerebrovascular pathology of the Spanish Society of Neurosurgery. The proposed recommendations should be considered as a general guide for the management of this pathological condition. However, they can be modified, even in a significant manner according to the circumstances relating each clinical case and the variations in the therapeutic and diagnostic procedures available in the center attending each patient.

KEY WORDS. Subarachnoid hemorrhage. Ischemia. Vasospasm. Aneurysm. Clip. Coil. Surgery. SENEC.

Recibido: 4-05-10. Aceptado: 6-12-10.

\section{Introducción}

La hemorragia subaracnoidea es una extravasación de sangre en el espacio subaracnoideo o leptomeníngeo. La causa más frecuente de hemorragia subaracnoidea es el traumatismo craneoencefálico. La hemorragia subaracnoidea primaria espontánea es con mayor frecuencia causada por la ruptura de un aneurisma cerebral, aunque existen otras causas como las malformaciones vasculares, tumores cerebrales, alteraciones de la pared vascular así como alteraciones de la coagulación. De un 15 a un $25 \%$ de los casos no se encuentra causa del sangrado constituyendo este grupo la hemorragia subaracnoidea idiopática cuyo pronóstico es mucho más benigno ${ }^{70,123,127,176,188}$. Esta guía trata sobre el manejo de los enfermos con hemorragia subaracnoidea aneurismática (HSA).

La HSA supone del 6 al 8\% de todas las enfermedades vasculares cerebrales agudas ${ }^{116}$, aunque su importancia radica en que afecta a pacientes de menor edad en general que el ictus isquémico y tiene una elevada morbi-mortalidad. Su incidencia no ha cambiado significativamente en los últimos años. Se han introducido nuevos métodos terapéuticos y cada vez más se usan protocolos que parecen haber mejorado la mortalidad general de esta enfermedad en las últimas décadas ${ }^{159}$.

En el año 2000 se publicaron en la revista Neurocirugía las guías elaboradas por el Grupo de Patología vascular de la SENEC (GVAS) ${ }^{178}$. Aunque el tratamiento endovascular ya era utilizado y recomendado en la anterior edición de la guía, la aparición de nuevas evidencias sobre sus indicaciones y resultados, así como la necesidad y modo de seguimiento de los enfermos tratados mediante esta técnica, y por otro lado, la aparición de unas nuevas guías internacionales $^{22}$, hizo que el GVAS decidiera revisar la versión anterior de estas guías.

Abreviaturas. AI: aneurismas incidentales. FLAIR: fluid attenuated inversion recovery. GVAS: grupo de Patología vascular de la SENEC. HSA: hemorragia subaracnoidea aneurismática.RM: resonancia magnética. TC: tomografía computarizada. WFNS: Federación Mundial de Sociedades Neuroquirúrgicas 


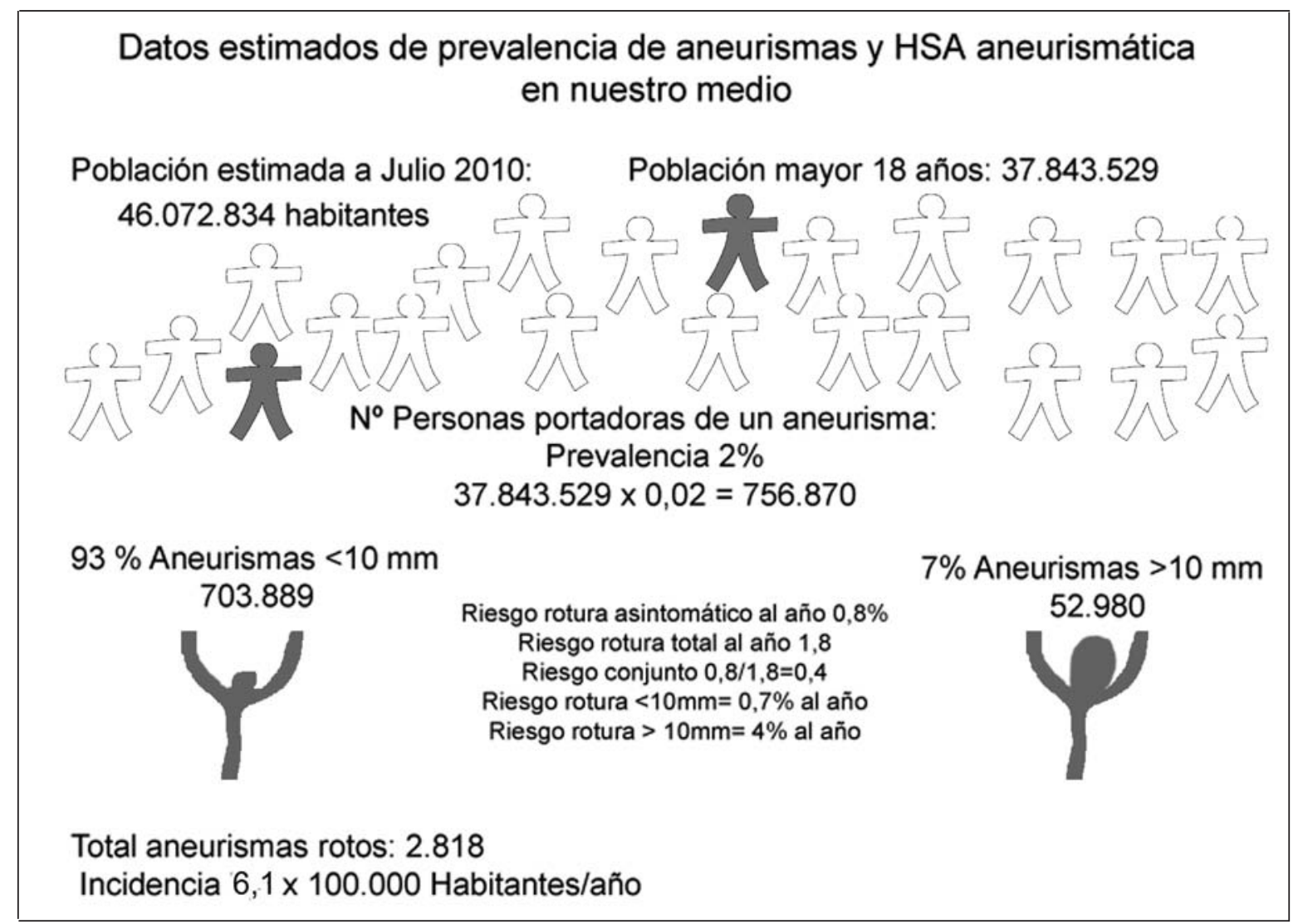

Figura 1. Aproximación al número de aneurismas y HSA que se producen en nuestro país aplicando los datos de Rinkel y cols.

\section{Epidemiología}

La hemorragia subaracnoidea aneurismática (HSA), es una enfermedad frecuente y potencialmente curable, si bien la morbimortalidad, considerada globalmente, es elevada. Hasta el $12 \%$ de los pacientes que la sufren no son adecuadamente diagnosticados o mueren antes de llegar al hospita ${ }^{94,103}$, y alrededor del $30 \%$ de los pacientes que llegan vivos al hospital fallecen en los primeros días ${ }^{63}$. Además, la morbilidad es significativa en el $50 \%$ de los supervivientes ${ }^{4,102,123,184}$. La mortalidad hospitalaria en nuestro país es similar a la descrita en estudios internacionales siendo del 26\%. Además hasta el 54\% de pacientes no alcanzan una recuperación completa según los datos de la base de datos multicéntrica del GVAS ${ }^{123}$. La incidencia de la HSA ha permanecido prácticamente estable a lo largo de los últimos 30 años, al contrario que otros tipos de accidentes cerebrovasculares ${ }^{102,170}$. La incidencia de ictus en general ha disminuido en la última década fundamentalmente por la disminución del hábito tabáquico y el mejor control de la hipertensión arterial. Dado que ambos factores también lo son de riesgo para la HSA se esperaría encontrar una disminución similar en el riesgo de HSA. Sin embargo, la disminución encontrada en un meta-análisis reciente de diferentes estudios epidemiológicos ha sido de tan sólo un $0,6 \%$ en las dos últimas décadas. Un meta-análisis de los estudios epidemiológicos publicados hasta la fecha demuestra que la incidencia de HSA oscila alrededor de 9/100.000 habs/año $0^{46 ; 134}$. En Finlandia ${ }^{46,63,169}$ o Japón ${ }^{86}$ por motivos desconocidos, se han reportado cifras que triplican esta incidencia. No existen datos epidemiológicos generales en nuestro país sobre la incidencia de esta enfermedad, aunque sí hay algún dato parcial referido a alguna comunidad autónoma ${ }^{37,150}$, siendo la incidencia calculada de la HSA menor que en otros países, ya que en estos estudios no supera los 5 casos anuales por cada 100.000 habitantes $^{37}$. La edad de presentación más frecuente en la HSA es alrededor de los 55 años, aumentando la incidencia al aumentar la edad. Así el porcentaje de pacientes en la base del GPVAS mayores de 55 años es del $66 \%$ siendo los mayores de 70 años el $17 \%$ del total ${ }^{123}$. En los estudios epidemiológicos analizados, se aprecia una mayor incidencia (entre 1.6 y 4.5 veces) en mujeres ${ }^{46,123,134,137}$ sobre todo a partir de los 55 años. En nuestro medio, al no existir un registro centralizado, es imposible conocer los datos exactos de la incidencia y prevalencia de HSA. Se ha realizado una aproximación al número teórico de 
aneurismas y HSA que se producen en nuestro país aplicando los datos de Rinkel y cols ${ }^{175}$ (Figura 1).

\section{Factores de riesgo y prevención}

Se han implicado diversos factores de riesgo para sufrir HSA entre ellos la hipertensión arterial, el hábito tabáquico, el alcohol, el consumo de drogas simpaticomiméticas como la cocaína ${ }^{57,172,205,206}$. Algunos estudios han descrito una mayor frecuencia de casos en relación a estaciones metereológicas, al aparecer una mayor frecuencia en invierno y primavera ${ }^{4,99}$, o en relación a cambios de presión ${ }^{35}$. Sin embargo, estos datos no se han corroborado en estudios nacionales ${ }^{16}$.

Parece que existe una predisposición familiar a la formación de aneurismas y por ello a sufrir HSA. Existen síndromes genéticos con mayor predisposición a presentar aneurismas como la poliquistosis renal autosómica dominante o la enfermedad de Ehlers-Danlos tipo IV ${ }^{6,34,55,190}$. Estos síndromes apoyan la posible existencia de una agregación familiar en la presencia de aneurismas. Diferentes estudios han encontrado una mayor frecuencia de aneurismas en gemelos de pacientes con aneurismas cerebrales o en familiares de afectos de HSA, sobre todo cuando hay más de un afecto en la familia. Así se ha calculado que tener 3 o más afectos en una familia triplica la probabilidad de encontrar otro individuo afectado en la familia ${ }^{7,32,33,66}$. Se han propuesto algunos protocolos de cribaje de familiares de afectos con resonancia magnética existiendo una importante rentabilidad diagnóstica cuando hay más de un caso afectado por hemorragia subaracnoidea, siendo además necesario repetir el estudio en el tiempo ${ }^{27,173,174}$. Sin embargo, no hay un consenso sobre la utilidad del cribado $^{3,22}$.

Por otro lado hay que tener en cuenta que los pacientes jóvenes tratados de aneurismas cerebrales presentan mayor predisposición a presentar con el tiempo nuevos aneurismas. Se ha calculado que estos enfermos tienen una frecuencia de formación de nuevos aneurismas de un 1 a $2 \%$ al año ${ }^{213,222}$. Este dato es importante para establecer el seguimiento de estos enfermos, aunque no se puede proponer ningún método o necesidad de cribado por el momento ${ }^{219,220}$.

La mejor prevención de la HSA consistiría en detectar aquellos pacientes con aneurismas cerebrales y tratarlos antes de que se produjera su ruptura. En estudios autópsicos y radiológicos se ha estimado que la prevalencia de aneurismas incidentales (AI) en la población general es alrededor de un $2 \%{ }^{100,175}$, aunque otros estudios han observado un incremento constante de la frecuencia de aneurismas con la edad oscilando entre el 1\% hasta los 30 años, y del 3 al 8\% entre los 40-70 años ${ }^{210}$. Con el aumento de las técnicas modernas de neuroimagen, cada vez es más frecuente tener que tomar una decisión ante un paciente portador de un aneurisma incidental. El tratamiento ideal en estos casos todavía es objeto de discusión debido a la selección de las poblaciones incluidas en los distintos estudios. King en 1994 realizó un estudio sistemático y un metaanálisis de las series más importantes publicadas hasta esa fecha ${ }^{117}$. En este estudio se apreció una morbilidad del $4.1 \%$ y una mortalidad del $1 \%$ en los pacientes tratados selectivamente. Sin embargo, no se pudieron identificar los factores de riesgo a tener en cuenta para indicar el tratamiento quirúrgico. En 1998 se han publicado los resultados obtenidos en el Estudio Internacional de Aneurismas Intracraneales Incidentales ${ }^{2}$. Este trabajo consta de un grupo de 1.449 pacientes en los que se analiza retrospectivamente la historia natural y un segundo grupo de 1.172 pacientes en los que se analiza prospectivamente la morbimortalidad del tratamiento. La incidencia media de sangrado es del $0.5 \% / a n ̃ o$. Esta cifra varía dependiendo del tamaño del aneurisma, apreciándose una incidencia de ruptura de $0.05 \%$ /año en aneurismas menores de $5 \mathrm{~mm}$ y sin historia de HSA previa; alrededor de un $1 \%$ /año en pacientes con aneurismas mayores de $10 \mathrm{~mm}$ y un $6 \%$ /año en aneurismas gigantes. En este análisis, el tamaño aneurismático (> $10 \mathrm{~mm}$ ) y la localización (vertebrobasilar) son factores predictivos independientes de ruptura. La morbimortalidad encontrada en el estudio prospectivo fue muy superior a la reportada hasta la fecha (13-15\% al año), siendo la edad el principal factor predictivo. Por lo tanto, concluyeron que el riesgo de rotura en aneurismas pequeños era muy bajo, excediendo el tratamiento quirúrgico el riesgo de rotura en estos casos. Sin embargo, se han publicado varias notas editoriales $^{12}$ rebatiendo estas conclusiones debido al sesgo introducido al comparar ambas poblaciones. Otros autores han identificado factores dependientes del individuo y del aneurisma como factores predictivos de rotura de un AI, entre los cuales destacan la edad superior a los 60 años, el sexo femenino, la localización en la circulación posterior, el tamaño mayor de $5 \mathrm{~mm}$ y si se trata de un aneurisma sintomático ${ }^{221}$. La decisión de tratar un AI se deberá individualizar en cada caso teniendo en cuenta la edad, tamaño y localización del aneurisma, patología de base y experiencia del equipo quirúrgico y endovascular ${ }^{17,21,91,92}$.

\section{Hemorragia subaracnoidea y embarazo}

La hemorragia subaracnoidea durante el embarazo, parto y puerperio es una situación de gravedad para la madre y el recién nacido. La mortalidad materna por HSA durante el puerperio es similar a la de la HSA en general ${ }^{212,215}$. La mortalidad fetal se aproxima al $17 \%$. No parece existir un incremento en la incidencia de HSA en el embarazo, parto o puerperio $^{207}$. No existe tampoco ninguna contraindicación para el embarazo en pacientes con cierto mayor riesgo de presentar aneurismas o HSA, ni tampoco existe una clara contraindicación para el parto vaginal, dado que éste no incrementa la incidencia de rotura aneurismática ${ }^{207}$. En el 
caso de una HSA por aneurisma en una mujer embarazada, se recomienda un tratamiento agresivo del aneurisma dado el riesgo de resangrado. Existe una teórica ventaja del tratamiento quirúrgico frente al endovascular. Durante la cirugía del aneurisma no debe utilizarse la hipotensión. Los agentes osmóticos no deben usarse dado el riesgo de hipoperfusión uterina e hiperosmolaridad fetal.

\section{Historia Natural}

Los mejores estudios de la historia natural de la HSA son los realizados en los años 60-70, ya que en aquella época la proporción de aneurisma rotos no tratados era muy superior a la de hoy día. En estos estudios se evidenció una alta mortalidad (alrededor del $60 \%$ en los primeros 6 meses) ${ }^{9,105,161,169,170,226}$. Al comparar estudios más recientes con otros previos, se ha comprobado que existe un leve descenso de la mortalidad y un aumento del porcentaje de pacientes con buena evolución final tras $\mathrm{HSA}^{38,64,102}$. Esta mejoría pudiera estar relacionada con un mejor conocimiento de la fisiopatología de esta enfermedad, y consiguientemente un tratamiento más adecuado de ésta, aunque el motivo de esta mejoría es incierto. No obstante, siguen existiendo grandes diferencias (hasta un 20\%) entre la supervivencia descrita en series hospitalarias y en series poblacionales ${ }^{223}$. Estas elevadas cifras de morbimortalidad apoyan que se adopten protocolos de tratamiento urgentes y eficaces, basados en un análisis sistemático de la literatura, con el fin de incluir al mayor número posible de pacientes con $\mathrm{HSA}^{22,60,139}$.

Diversos factores influyen en la evolución de los pacientes con $\mathrm{HSA}^{126}$. Entre ellos destaca la gravedad del sangrado inicial por el importante peso que tiene en la evolución final de la enfermedad ${ }^{47,125,126}$. La hemorragia produce importantes y profundas reducciones del flujo sanguíneo cerebral asociadas a un incremento agudo de la presión intracraneal que desencadenan un daño isquémico que se puede mantener más allá de los primeros momentos del sangrado ${ }^{84,144,162}$. Estos procesos aunque cada vez más reconocidos no han encontrado todavía un tratamiento efectivo. Por supuesto la existencia de comorbilidad como en otros cuadros graves dificultará el tratamiento y empeorará el resultado final del enfermo ${ }^{43}$. Por otro lado hay factores relacionados con la localización y morfología del aneurisma responsable del sangrado que también influyen en el pronóstico tales como el tamaño del aneurisma, su localización en la circulación posterior y posiblemente su morfología ${ }^{191}$. Por otro lado, parece cada vez más evidente que existen factores relacionados con la institución que realiza el tratamiento tales como la disponibilidad de tratamiento endovascular así como el volumen de pacientes que $\operatorname{trata}^{25,107}$.

A pesar de que el tratamiento precoz de los aneurismas está cada vez más extendido, el resangrado continúa siendo una causa importante de mortalidad y morbilidad. El riesgo de resangrado con tratamiento conservador de los aneurismas es de hasta un 30\% en el primer mes estabilizándose posteriormente en un $3 \%$ al año ${ }^{225}$. El riesgo de resangrado aumenta en enfermos con presión arterial elevada, mal grado clínico y en aquéllos en los que el periodo entre el diagnóstico y el tratamiento es mayor. El resangrado es la principal causa de mortalidad tratable y debe ser evitado.

\section{Clínica y diagnóstico}

Antes de la ruptura "mayor" de un aneurisma, pueden ocurrir síntomas premonitorios ("cefalea centinela") hasta en un $45 \%$ de los $\operatorname{casos}^{20}$. El síntoma más frecuente es una cefalea brusca, debido probablemente a un pequeño sangrado aneurismático, que se diagnostica mal en un $12 \%$ de los $\operatorname{casos}^{23}$. La ruptura mayor del aneurisma sucede a la cefalea centinela entre 1 y 6 semanas. La HSA es una emergencia médica, siendo esencial su diagnóstico precoz (hasta un $20 \%$ son mal diagnosticadas inicialmente) e ingreso para tratamiento del paciente en un medio adecuado. Siempre se debe sospechar la presencia de HSA cuando existe una cefalea intensa ("la más fuerte de mi vida"), de aparición brusca, pudiendo ir seguida de alteración en el sensorio, náuseas, vómitos, rigidez de nuca y déficits focales incluyendo parálisis de pares craneales. Los diagnósticos incorrectos más frecuentes son: infección viral, migraña, cefalea hipertensiva, espondiloartrosis cervical, etc. Es importante estimar el grado clínico de cada paciente ya que existe una buena correlación entre la evolución final y el grado clínico inicial. Las escalas de evaluación clínica dan idea del efecto inicial de la hemorragia y de los efectos fisiopatológicos que suceden en el comienzo de la enfermedad. A lo largo de los años se han propuesto multitud de clasificaciones ${ }^{77,125,163}$. Aunque no existe una escala perfecta, hoy día las más validadas son la de Hunt y Hess ${ }^{96}$ y la propuesta por la Federación Mundial de Sociedades Neuroquirúrgicas (WFNS), basada en la escala de coma de Glasgow cuya puntuación desglosada debe quedar también registrada $^{1}$ (Tabla 1).

La TC es la prueba más sensible en el diagnóstico de la HSA; siempre se debe practicar lo antes posible después de la sospecha clínica de HSA ya que con el paso de los días pierde sensibilidad al irse lisando la sangre depositada en el espacio subaracnoideo. Únicamente un $2-5 \%$ de los pacientes con HSA tienen una TC normal en el primer día tras el sangrado, presentando una sensibilidad en las primeras 12 horas de entre el 98 y $100 \%$, pasando a un $93 \%$ a las 24 horas y el $57 \%$ a los seis días ${ }^{149,185,197,211}$. Además del diagnóstico, la TC aporta una valiosa información sobre la extensión y localización de la sangre, presencia de 
Tabla 1

Escalas más utilizadas en relación a la HSA

\section{GLASGOW COMA SCALE (GCS)}

La escala de coma de Glasgow representa la suma de la valoración de tres respuestas: repuesta ocular + respuesta verbal + respuesta motora.

Respuesta ocular

Espontánea 4

Al habla 3

Al dolor 2

No respuesta 1

$\begin{array}{ll}\text { Respuesta verbal } \\ \text { Orientada } & 5 \\ \text { Confusa } & 4 \\ \text { Incoherente } & 3 \\ \text { Incomprensible } & 2 \\ \text { Sin respuesta } & 1\end{array}$

\section{ESCALA DE HUNT Y HESS}

\section{Respuesta motora}

Obedece órdenes 6

Localiza 5

Retirada 4

Flexión anormal 3

Extensión anormal 2

0- Aneurisma no roto.

I- Asintomático o cefalea leve y/o rigidez leve de nuca.

Ia- Ausencia de reacción meníngea o cerebral, pero presencia de un déficit neurológico fijo.

II- Parálisis de pares craneales, cefalea moderada o intensa o/y meningismo moderado severo. Ill Déficit focal leve, letargia o confusión.

IV- Estupor, hemiparesia moderada o severa, o rigidez precoz de descerebración.

V- Coma profundo, rigidez de descerebración, aspecto moribundo.

\section{WORLD FEDERATION OF NEUROLOGICAL SURGEONS (WFNS)}

I- GCS 15, sin déficit motor.

II- GCS 14-13, sin déficit motor.

III- GCS 14-13, con déficit motor.

IV- GCS 12-7, con o sin déficit motor.

V- GCS 6-3, con o sin déficit motor.

\section{ESCALA DE FISHER}

1- No se detecta sangre en la TC craneal.

2- Capas difusas o verticales $<1 \mathrm{~mm}$ de grosor.

3- Coágulos localizado o/y capa vertical $>1 \mathrm{~mm}$ de grosor.

4- Coagulo intracerebral o intraventricular con HSA difusa o sin ella. 
dilatación ventricular, hematomas intraparenquimatosos, etc. La cuantificación del depósito hemático en el espacio subaracnoideo es muy difícil de determinar. La escala más utilizada hoy día es la de Fisher ${ }^{61}$, aunque se han propuesto otras que tienen en cuenta el volumen de sangrado intraventricular ${ }^{41}$. Dado que la sensibilidad de la TC no es absoluta, la PL debe realizarse cuando existen dudas de la presencia de sangre en la TC inicial (TC normal o retraso en la referencia a un centro hospitalario). La resonancia magnética (RM) ha sido utilizada también en el diagnóstico de sospecha de HSA, fundamentalmente con la aplicación de imágenes FLAIR (fluid attenuated inversion recovery) ${ }^{15,59,136}$. También se ha usado en el diagnóstico de la causa del sangrado pero esta técnica ha sido superada por la mayor disponibilidad y rapidez por el TC helicoidal o angio TC. Esta prueba alcanza una sensibilidad diagnóstica muy alta, próxima al $83 \%$, y ha sido utilizada ya por muchos autores como única prueba previa a la cirugía en los casos en los que no está justificado el retraso en la cirugía por la angiografía ${ }^{24,26,48,72,73-75,227}$. Tiene peor resolución para aneurismas pequeños ${ }^{11,44}$ pero el angio TC es capaz de definir mejor la presencia de calcificaciones en el aneurisma, trombosis del mismo, y relación del aneurisma con referencias óseas o localización dentro de un hematoma. Por ello, la angio-TC puede y es considerada hoy en día por muchos grupos como la primera prueba diagnóstica de elección para la detección de aneurismas en pacientes con HSA $\mathrm{y}$, con ello, el método sobre el que decidir el tratamiento idóneo para la exclusión del aneurisma.

Sin embargo, la prueba estándar para el diagnóstico de aneurismas responsable de HSA sigue siendo la angiografía cerebral y, por lo tanto, aquellos pacientes con HSA y angio-TC negativo deben ser sometidos a arteriografía de cuatro vasos para descartar la presencia de patología vascular subyacente. Esta prueba se deberá realizar lo antes posible tras la hemorragia (no se aconseja realizar en las primeras 6 horas del sangrado, pues parece que aumenta el riesgo de resangrado ${ }^{98}$, dependiendo de la disponibilidad del Servicio de Radiología. Con esta prueba se aprecian las características anatómicas del aneurisma y de los vasos del polígono de Willis, así como datos fundamentales en la planificación del tratamiento quirúrgico. Así mismo, se puede hacer una valoración del estado de la circulación cerebral (predominancias arteriales, flujo cruzado, etc.). El grado de vasoespasmo angiográfico se clasifica según Fisher ${ }^{61}$.

\section{Tratamiento HSA}

No existe aún un consenso general entre diferentes autores para tratar los diferentes aspectos de esta enfermedad y los protocolos de manejo han cambiado considerablemente a lo largo del tiempo, variando entre distintos centros y países.
Recientemente se tiende a adoptar en la mayoría de los centros un protocolo de manejo más uniforme, especialmente diseñado para mejorar la evolución global de la enfermedad y no sólo de los casos seleccionados para la cirugía. Para ajustarse con éxito a estos protocolos, es necesaria la estrecha colaboración entre neurólogos, neurocirujanos, intensivistas y neurorradiólogos intervencionistas.

Los objetivos fundamentales para un correcto tratamiento de esta enfermedad son:

1. Diagnóstico precoz: en el $20 \%$ de los casos no se diagnostica adecuadamente la primera hemorragia. Es esencial el traslado inmediato a un centro hospitalario con servicios de Neurocirugía, UVI, y Neurorradiología. El neurocirujano sería el responsable de coordinar a los distintos especialistas integrados en el manejo de la HSA.

2. Prevención del resangrado: mediante cirugía y/o embolización.

3. Estabilización del paciente crítico en UVI, con el fin de intentar que la mayoría de los casos sean potencialmente tratables, mediante cirugía y/o embolización.

4. Prevención y tratamiento agresivo de la isquemia cerebral, especialmente en los casos en los que ya se ha ocluido el aneurisma.

A pesar de que el ictus isquémico ha recibido atención por parte de las autoridades sanitarias en cuanto a su tratamiento agudo y su traslado a centros con Unidades de Ictus, la HSA no ha recibido tanta atención. Sin embargo, buen número de pacientes con HSA podrían beneficiarse de recibir de forma inicial un tratamiento y traslado similar. Es evidente que sería deseable que se instaurara algún mecanismo para detectar aquellos enfermos con alta sospecha de HSA y que éstos fueran remitidos a centros donde pudieran ser tratados, evitando traslados secundarios $^{23}$. El manejo inicial de un enfermo con ictus isquémico o hemorrágico debe ser similar. Ha de tenerse en cuenta su situación neurológica, dando especial importancia al nivel de conciencia. Por ello, como ya se ha comentado, la evaluación inicial y la monitorización de la situación neurológica del enfermo debe ser realizada obteniendo la puntuación en la escala de coma de Glasgow (GCS) y el WFNS del enfermo. Es fundamental como en cualquier otro cuadro grave asegurar una adecuada ventilación a través del mantenimiento de la vía aérea, una adecuada oxigenación y perfusión. Existe potencial de deterioro neurológico y por lo tanto de incapacidad para mantener una adecuada ventilación y por ello los enfermos con alteración del nivel de conciencia deberán ser intubados si es necesario. Por todo ello, y debido a la necesidad de una estrecha vigilancia de los enfermos, su exploración neurológica y constantes, consideramos que todos los enfermos que sufren HSA deben ser manejados en una unidad de cuidados intensivos. 


\section{A. Medidas generales}

- Monitorización de los pacientes con HSA. Los pacientes con HSA tienen un riesgo importante de deterioro neurológico. Por ello es fundamental realizar una monitorización estrecha de su situación previa al tratamiento del aneurisma.

- Valoración periódica del GCS y tamaño pupilar.

- Electrocardiograma (ECG).

- Frecuencia cardiaca (FC).

- Saturación de oxígeno.

- Tensión arterial invasiva o no, según el grado de severidad.

- Diuresis horaria (sondaje vesical).

- Presión venosa central (PVC): Catéter vía central.

- Control de la temperatura.

- Glicemias capilares.

- Presión intracraneal (PIC) y presión de perfusión cerebral (PPC) en pacientes con un GCS inferior a 9. La medición puede realizarse mediante un sensor intraparenquimatoso o asociado a un drenaje ventricular externo.

- Asegurar una ventilación y oxigenación adecuada.

- PO2 alrededor de $80-100 \mathrm{mmHg}$ o saturación de oxígeno igual o superior a 95\%, y pCO2 alrededor de 35$45 \mathrm{mmHg}$.

- Valorar si el paciente precisa intubación según el estado neurológico (GCS inferior o igual a 8) o la función respiratoria.

- Control de la HTA

- Se considera que la presión arterial media (PAm) óptima para mantener una buena perfusión cerebral es de 90-110 mmHg. Es muy importante evitar tanto la hipertensión como la hipotensión arterial.

- Las cifras elevadas de presión arterial (PA) pueden ser reactivas al dolor, hipoxia, isquemia cerebral o hipertensión intracraneal, por lo que sólo se iniciará tratamiento antihipertensivo si las cifras persisten altas tras haber corregido estos factores.

- Será necesario un control estricto de la PA, ya que una caída importante de la PA puede originar una disminución de la perfusión cerebral y empeorar o precipitar la isquemia cerebral.

- Un fármaco a utilizar es el labetalol, que por su efecto $\beta 1$-bloqueante selectivo causará una reducción del gasto cardíaco, sin provocar vasoconstricción cerebral.

- Se utiliza también nimodipino (calcioantagonista) que además previene el vasoespasmo cerebral.

- Los diuréticos están contraindicados al causar depleción del volumen intravascular.

- Profilaxis de ulcus por estrés

- Profilaxis del ulcus de estrés con inhibidores de la bomba de protones.

- Profilaxis de la TVP

- Se deben colocar medias de compresión neumática intermitente las primeras $48 \mathrm{~h}$ (según analítica y estado clínico del paciente). Si el tratamiento aneurismático es quirúrgico se prolongará $48 \mathrm{~h}$ más. Posteriormente se iniciará profilaxis con heparinas de bajo peso molecular (Enoxiparina, Bemiparina o HiboR $3500 \mathrm{U} / 24 \mathrm{~h} / \mathrm{sbc}$ ).

- Tratamiento analgésico

- La pauta inicial se realizará con Paracetamol $1 \mathrm{gr} / 8 \mathrm{~h} /$ ev y/o Dexketoprofeno $50 \mathrm{mg} / 8 \mathrm{~h} / \mathrm{ev}$ (no prolongando este último más de 3 días por el riesgo de insuficiencia renal).

- En casos de cefalea refractaria valorar Tramadol $100 \mathrm{mg} / 8 \mathrm{~h} / \mathrm{ev}$ o Meperidina $1 \mathrm{mg} / \mathrm{kg} / 8 \mathrm{~h} / \mathrm{sc}$, asociando profilaxis antiemética con Metoclopramida $1 \mathrm{amp} / 8 \mathrm{~h} / \mathrm{ev}$.

- La Dexametasona en bolus de $8 \mathrm{mg} / \mathrm{ev}$ se utilizará en caso de cefalea persistente secundaria a síndrome meníngeo (náuseas y vómitos).

- Tratamiento sedante

- Se puede utilizar como sedante el cloracepato dipotásico (Tranxilium $\left.{ }^{\circledR}\right) 20 \mathrm{mg} / 12 \mathrm{~h} / \mathrm{ev}$ en pacientes no intubados como ansiolítico, teniendo en cuenta que es muy importante poder hacer una valoración del estado neurológico del paciente sin que haya fármacos que interfieran. Se debe evitar una sedación profunda.

- En Unidades de Críticos, en pacientes despiertos, se puede utilizar remifentanilo (Ultiva ${ }^{\circledR}$ ) endovenoso a dosis bajas (0.02-0.05 $\mathrm{mcg} / \mathrm{kg} / \mathrm{min})$.

- En caso de pacientes con ventilación mecánica se suele utilizar propofol y/o remifentanilo como hipnótico y analgésico respectivamente, de mantenimiento por su corta vida media, ya que permite una rápida valoración neurológica del paciente tras su retirada.

- Control continuo del ECG

- Sirve para detectar precozmente alteraciones del ritmo, infartos, etc, ya que la HSA puede estar asociada a descarga masiva simpática.

- Control horario de PVC

- Permite mantener un balance hídrico adecuado, evitando siempre la hipovolemia.

- Tratamiento antiemético

- Se utiliza en caso de náuseas, vómitos o retención gástrica con Metoclopramida (Primperán ${ }^{\circledR}$ ) 1ampolla/8h/ev.

- Sueroterapia con líquidos isotónicos o hipertónicos

- El control de líquidos y electrolitos debe ser muy cuidadoso, evitando siempre la hipovolemia, deshidratación y 
hemoconcentración. La administración de líquidos puede reducir la viscosidad sanguínea y mejorar la perfusión cerebral.

- Se deben tomar precauciones para evitar la hiponatremia y la sobrecarga de agua libre que pueden exacerbar el edema cerebral. Se recomienda administrar sueros isotónicos.

- La hiponatremia en la HSA no suele ser debida a un síndrome de secreción inadecuada de ADH (más frecuente en el traumatismo craneoencefálico) sino a una natriuresis excesiva con hipovolemia o síndrome pierde sal y que se debe corregir con suero hipertónico y expansores del plasma.

\section{- Controlar la temperatura horaria}

- La hipertermia aumenta el flujo y volumen sanguíneos cerebrales, lo que provoca un aumento de la PIC. El tratamiento debe ser agresivo e inmediato y se debe encontrar el foco infeccioso que suele ser la principal causa.

- Controlar las glicemias

- Mantener glicemias entre 110 y $150 \mathrm{mmol} / \mathrm{ltr}$ con insulina rápida subcutánea endovenosa si se precisa ${ }^{129}$. No es recomendable el control estricto dirigido a valores de normoglucemia entre 80 y $110 \mathrm{mmol} / \mathrm{ltr}$ por el elevado riesgo de hipoglucemia.

-Tratamiento y prevención de crisis comiciales

- Las crisis comiciales ocurren en el contexto de la HSA con una frecuencia muy variable, entre el $3-30 \%$ y pueden contribuir al sangrado del aneurisma cerebral.

- No hay unanimidad en el uso de antiepilépticos como prevención de crisis comiciales, pero sí cuando el paciente presenta una crisis para que no reaparezcan.

\section{B. Tratamiento médico específico}

Los objetivos fundamentales del tratamiento médico de la HSA son:

\section{1.- Prevención del resangrado:}

A). Reposo en cama. Antiguamente se proponía como alternativa al tratamiento quirúrgico para la prevención de resangrado. En el Estudio Cooperativo se demostró su ineficacia en la prevención de resangrado.

B). Control tensión arterial. La presión arterial debe ser monitorizada. Parece claro que el resangrado puede venir precedido o coincidir con picos hipertensivos. Aunque la disminución de la presión arterial no ha conllevado una disminución de la frecuencia del resangrado, deben evitarse oscilaciones bruscas.

C). Antifibrinolíticos ${ }^{62,111,155}$. Reducen hasta un $45 \%$ el riesgo de sangrado, pero no mejoran la evolución final ya que se aumenta la incidencia de isquemia e hidrocefalia secundarias. Sin embargo, estos estudios se realizaron hace más de 10 años, antes de la introducción de la nimodipina y del conocimiento de la prevención de la hipovolemia en el desarrollo de la isquemia. Por lo tanto, los antifibrinolíticos podrían ser hoy día útiles usados concomitantemente con estos otros métodos que disminuyan el riesgo de isquemia ${ }^{40,90,114}$ en pacientes con bajo riesgo de isquemia, en los que se decide retrasar la intervención, o también en ciclos de corta duración previa a una cirugía en los primeros días tras el sangrado. En un estudio prospectivo randomizado en el que se utilizó ácido tranexámico a altas dosis en el momento del diagnóstico se consiguieron reducir los resangrados y el porcentaje de pacientes con mala evolución ${ }^{90}$.

\section{2. - Prevención de la aparición de isquemia cerebral.}

A). Evitar hipovolemia, administrando suficiente cantidad de fluidos intravenosos. Nunca se deberá restringir líquidos en caso de hiponatremia. Se ha demostrado que esta situación está producida por una eliminación anormal de sal por orina, por lo que hay que restituirla adecuadamente.

B). Bloqueantes del calcio, varios estudios prospectivos randomizados y dos revisiones sistemáticas con metaanálisis de todos los estudios publicados, demuestran la utilidad de los bloqueantes del calcio, especialmente la nimodipina tanto en forma oral como intravenosa ${ }^{18,56,166,171}$. En estos trabajos se aprecia una prevención en la aparición de los déficits isquémicos de un $33 \%$, una reducción de mala evolución final de un $16 \%$ y reducción global de la mortalidad de un $10 \%$.

\section{Tratamiento del aneurisma}

\section{Tratamiento quirúrgico}

El fin primordial del tratamiento quirúrgico es evitar el resangrado, con la adecuada colocación de un clip en el cuello aneurismático. No se recomienda el recubrimiento ("coating") o el empaquetamiento ("wrapping") del saco, pues no reduce significativamente el riesgo de resangrado. El "trapping" del aneurisma o la ligadura de carótida pueden estar indicados en determinadas ocasiones.

Desde la introducción de las técnicas microquirúrgicas hace más de 30 años, el abordaje de estas lesiones ha alcanzado un alto grado de perfección técnica y parece difícil que esta pueda mejorarse $\mathrm{e}^{195,203,227}$. Hasta hoy día, la cirugía se ha considerado el tratamiento de elección en la HSA, si bien en el mejor de los casos (referencia inmediata, cirugía precoz), sólo se podrían llegar a operar alrededor del $60 \%$ de los pacientes con aneurismas ${ }^{64}$; aunque es difícil comparar los resultados de las distintas series publicadas en la literatura, ya que incluyen poblaciones muy diferentes, por lo tanto, las cifras de mortalidad serán muy diferentes si el estudio 
es poblacional, hospitalario o si sólo incluye a los pacientes operados $^{68,89,101,147,223}$. Siempre ha existido una controversia sobre cuál es el mejor momento para intervenir a un paciente

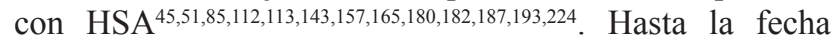
sólo existen dos estudios prospectivos, randomizados ${ }^{88,165}$ que demuestren el beneficio de la cirugía precoz (0-3 días) respecto a la tardía(>7-10 días). Ohman reportó que a los 3 meses de la cirugía, el $91.5 \%$ de los pacientes operados en los 3 primeros días eran independientes, con una mortalidad del $5.6 \%$. Por otro lado, $80 \%$ de los operados tardíamente (> 10 días) eran independientes, con una mortalidad de $13 \%$. Heiskanen en el otro estudio randomizado en pacientes con hematoma intraparenquimatoso secundario a rotura de aneurisma, evidenció una mortalidad del $80 \%$ para los tratados conservadoramente frente a un $27 \%$ de los operados. En el Estudio Cooperativo de los aneurismas (no randomizado) ${ }^{112,113}$ sobre 3.521 pacientes con HSA, se operaron 2.922 (83\%) 53\% de éstos en los 3 primeros días, con una morbilidad quirúrgica del $8 \%$ y una mortalidad global de $26 \%$. Sáveland ${ }^{189}$, publicó un estudio prospectivo realizado a lo largo de un año, en el que se incluyeron 325 pacientes no seleccionados con HSA que ingresaron en 5 de los 6 servicios suecos de neurocirugía. En dicho estudio, se intervinieron 276 (85\%) pacientes, de éstos 170 (62\%) en los primeros 3 días, obteniéndose una morbilidad quirúrgica del $7 \%$ y una mortalidad global del $21 \%$. Aunque hoy día se recomienda cirugía precoz (0-3 días) en aquellos pacientes en buen grado clínico (I-III de la WFNS) y aneurismas no complejos, el día de la cirugía por sí mismo, no tiene valor predictivo. En la decisión también influyen otros factores como la edad, enfermedades concomitantes, localización, tamaño, complejidad del aneurisma y disponibilidad de medios. Un factor decisivo a tener en cuenta en el momento de la decisión de intervención precoz o demorada, es el mal grado clínico inicial (Grados IV-V de la WFNS). Antiguamente estos pacientes eran manejados conservadoramente o se practicaba cirugía tardía en aquellos que sobrevivían; varios autores $14,50,97,119,128,130,145,181,194$ han preconizado un tratamiento médico y quirúrgico agresivo en estos casos, demostrando una mejor evolución final, incluso en pacientes en grado $\mathrm{V}$ obteniendo una buena recuperación o incapacidad leve entre $20-40 \%$ del total de pacientes tratados.

Durante la intervención se debe evitar la hipotensión (TA sistólica $<60 \mathrm{mmHg}$ ). Durante la disección arterial puede ser necesario el "clipaje" temporal de alguno de los vasos de asiento del aneurisma ${ }^{164,183}$. No está aún determinado el tiempo máximo seguro de oclusión, pero no es conveniente sobrepasar los 20 minutos. La oclusión temporal intermitente parece que ofrece menos riesgos de isquemia, aunque todavía no están bien definidos los tiempos de oclusión. En algunos aneurismas proximales paraclinoideos puede recurrirse a la oclusión temporal de la carótida interna cervical, clipaje transitorio distal al aneu- risma e incluso vaciado carotídeo retrógrado para facilitar la disección y clipaje definitivo del cuello aneurismático ${ }^{71}$.

Como se ha comentado previamente los aneurismas pueden ser tratados mediante la oclusión de la arteria portadora, aunque esta oclusión conlleva riesgo de isquemia. Este procedimiento se reserva para aneurismas no tratables mediante otras técnicas y es un tratamiento de elección en el caso de aneurismas disecantes y en ampolla o blebs ${ }^{5,19,131,141}$ o aneurismas que no pueden ser tratados mediante ninguna otra técnica disponible $e^{93}$. La presencia o no de isquemia tras la oclusión puede ser predicha mediante un test de oclu$\operatorname{sión}^{201}$. Dicho test se realiza mediante el inflado durante la angiografía de un balón que ocluye el vaso. Durante esta oclusión se ha de monitorizar la función neurológica, bien mediante la exploración neurológica o mediante algún método electrofisiológico. Si no se producen déficits tras cierta hipotensión en principio se podría ocluir la arteria sin que se produjeran déficits neurológicos. Hoy en día se utiliza también el retraso en la fase venosa de la angiografía para predecir la probabilidad de isquemia. Cuando el test de oclusión es positivo, deberá realizarse un by-pass extraintracraneal para llevar a cabo la oclusión de forma segura. Este algoritmo de tratamiento se está usando cada vez con mayor frecuencia en nuestro país ${ }^{49,124,177}$.

Una teórica ventaja de la cirugía es el lavado de sangre cisternal, con lo que teóricamente se puede reducir la incidencia de isquemia postoperatoria; esta premisa no se ha podido demostrar, incluso se ha visto que con el lavado agresivo de las cisternas aumenta el riesgo quirúrgico ${ }^{228}$.

\section{Tratamiento endovascular}

A principios de los años 90 se introdujo la embolización endovascular con espirales ("coil") de platino (GDC) $)^{81-83}$; inicialmente se utilizaba fundamentalmente en aneurismas complejos en los que se preveía un alto riesgo quirúrgico, o en pacientes que habían rechazado la cirugía, o en aquéllos en los que ésta había fallado. Poco a poco esta técnica se ha refinado y se ha extendido considerablemente, ampliándose sus indicaciones llegando a superponerse a las de la cirugía. La comparación de los resultados obtenidos con una u otra técnica es imposibles de realizar debido a la heterogeneidad de las poblaciones incluidas en cada serie. Hasta la fecha sólo un estudio prospectivo randomizado compara ambos procedimientos ${ }^{118}$, encontrando una buena evolución a los 3 meses en el $79 \%$ de los pacientes operados y un $81 \%$ en los embolizados, con una mortalidad del $11 \%$ y del $12 \%$ respectivamente. Viñuela ${ }^{218}$ en un Estudio Cooperativo de 403 pacientes con HSA y aneurismas de difícil acceso quirúrgico, embolizados en los primeros 15 días de la hemorragia encontró un $9 \%$ de morbilidad, y un $6 \%$ de mortalidad globales ( $2 \%$ secundaria al procedimiento); $6 \%$ de los pacientes requirieron tratamiento quirúrgico tras una 
embolización incompleta. En los aneurismas pequeños con cuello pequeño se obtuvo una oclusión prácticamente total en el $92 \%$, pero sólo un $30-50 \%$ de los pacientes con cuellos grandes o aneurismas gigantes tuvieron una oclusión satisfactoria.

Las complicaciones relacionadas con el procedimiento endovascular son la perforación del aneurisma que ocurre en torno a $2,4 \%$ de los casos y complicaciones isquémicas, bien por embolismo arterial u oclusión o trombosis del vaso portador del aneurisma en un $9 \%{ }^{30}$. Sin embargo estas complicaciones alteran con poca frecuencia el pronóstico marcado en el enfermo por el nivel de conciencia al ingreso o la gravedad de la hemorragia ${ }^{47,126}$.

La eficacia del tratamiento de los aneurismas viene marcado por dos aspectos: disminuir el riesgo de resangrado y conseguir un tratamiento definitivo del aneurisma, es decir, conseguir su exclusión completa de la circulación cerebral. El riesgo de resangrado en los aneurismas embolizados disminuye hasta un 0,9 a $2,9 \%$, aunque otros estudios han estimado un riesgo de un $1,4 \%$ al año de re-ruptura $^{36,115,146,200}$. Parece que uno de los factores más importantes a la hora de producirse una recurrencia o hemorragia tras el tratamiento endovascular es el tamaño y la forma del aneurisma tratado. Para los aneurismas mayores de 2 $\mathrm{cm}$ el resangrado es frecuente alcanzando un $33 \%$ en un estudio $^{80}$. La recurrencia de los aneurismas también es mayor en aneurismas grandes, fundamentalmente porque la frecuencia de tratamientos incompletos es mayor ${ }^{122,200}$. Cuando el tratamiento es incompleto la frecuencia de crecimiento del resto del aneurisma es alta, alcanzando cifras de hasta el $49 \%$. El tratamiento es con poca frecuencia completo en series globales de aneurismas, siendo este el resultado hasta en un 55\% de los casos. El tamaño del aneurisma y del cuello parecen tener un papel importante en el resultado ${ }^{151,199}$. Los peores resultados se obtienen en cuellos anchos y mayores tamaños ${ }^{39,58}$. El riesgo de recurrencia del aneurisma es también alto en aneurismas tratados de forma completa siendo factores de riesgo para su crecimiento el mayor tamaño del aneurisma o su situación con respecto al flujo sanguíneo como la cerebral media o la basilar ${ }^{39}$.

Aunque el seguimiento de los aneurismas embolizados se ha llevado a cabo tradicionalmente mediante la angiografía, parece que la RM craneal puede servir como una alternativa a la angiografía, dejando únicamente la angiografía para el caso de relleno evidente en la $\mathrm{RM}^{54}$. La necesidad de seguimiento de los enfermos tratados mediante embolización es evidente, y por ello recomendamos la realización de pruebas de control a largo plazo a estos enfermos.

Aunque la frecuencia de recanalizaciones y crecimiento de aneurismas tratados mediante tratamiento endovascular continua siendo alta, nuevos avances técnicos probablemente determinarán mejores resultados a este respecto. Por otro lado parece evidente que el uso de esta tecnología ha hecho disminuir la mortalidad de los enfermos con HSA y por ello debe ser utilizada en aquellos casos en los que se consigan mejores resultados ${ }^{142}$.

\section{Indicaciones de tratamiento}

El tratamiento de los aneurismas cerebrales debe ser realizado por un equipo con experiencia constituido por neurocirujanos formados en el tratamiento de patología vascular cerebral e intervencionistas con experiencia en la realización de angiografías cerebrales y en el tratamiento de estas lesiones. El tratamiento debe ser por tanto realizado en centros que dispongan de ambos especialistas trabajando de forma conjunta ${ }^{22}$. Cada vez es más evidente que el incremento de la experiencia en el tratamiento de esta patología mejora los resultados. Además los centros que incluyen el tratamiento endovascular tratan antes a los enfermos y tienen mejores resultados en general. La recomendación en este sentido es concentrar el tratamiento de estos enfermos en centros que dispongan de ambas técnicas ${ }^{43,153}$, aunque el efecto de la concentración del tratamiento es menor en los aneurismas rotos. Deberá asimismo establecerse la mejor indicación dependiendo de las características del paciente, su estado clínico y comorbilidad, las características del aneurisma a tratar y la experiencia propia del centro. Aunque de la revisión de la literatura no se pueden extraer indicaciones estrictas, si se pueden sugerir algunas indicaciones generales. El estudio ISAT, en el que se incluían enfermos que podían ser tratados tanto por tratamiento endovascular como quirúrgico, demostró que aunque la mortalidad era similar en ambos ramos de tratamiento, la morbilidad asociada al tratamiento endovascular era menor. Así pues, el tratamiento endovascular debe ser utilizado cuando los resultados con ambas técnicas sean juzgados equivalentes por el equipo encargado del tratamiento ${ }^{146}$. Algunos estudios neuropsicológicos y de calidad de vida en nuestro medio en pacientes tratados con una u otra modalidad terapéutica aprecian una mínima y escasa diferencia a favor de la embolización ${ }^{114,168}$. Aunque hay aneurismas que pueden ser tratados de forma general mediante ambas técnicas, parece que existen casos en los que un determinado tratamiento es más favorable. Por ello se pueden hacer las siguientes recomendaciones.

Las indicaciones principales de la embolización, aunque éstas están en continua evolución son:

1.- Fallo de la exploración quirúrgica.

2.- Mal grado clínico inicial.

3.- Mala condición médica.

4.- Aneurismas complejos con alto riesgo quirúrgico.

5.- Aneurismas de circulación posterior.

6.- Inoperabilidad por consideraciones anatómicas.

7.- Rechazo cirugía. 
La embolización no está indicada o sus resultados van a ser inferiores en:

1.- Aneurismas con cuellos anchos. Se definen como aneurismas de cuello ancho aquéllos con un cuello mayor a $4 \mathrm{~mm}$ o bien una relación entre el cuello del aneurisma y el diámetro mayor del aneurisma mayor de 2 .

2.- Hematomas intraparenquimatosos que requieren evacuación urgente.

3.- Aneurismas gigantes y grandes de fácil acceso neuroquirúrgico (circulación anterior).

4.- Aneurismas de cerebral media. En esta localización los resultados quirúrgicos suelen ser buenos. Además la disposición y localización del aneurisma y las ramas de la cerebral media hacen que el tratamiento de estos aneurismas sea complejo desde el punto de vista endovascular. Esta localización está relacionada con una mayor frecuencia de recanalización y crecimiento ${ }^{29,39}$.

5.- Aneurismas muy pequeños $(<2 \mathrm{~mm})$.

En el caso de aneurismas múltiples ${ }^{67,216}$ se comenzará con el aneurisma con más posibilidades de haberse roto, para lo cual se valorarán los siguientes signos:

- Distribución de sangre en TC inicial.

- Aneurismas de mayor tamaño.

- Bordes lobulados o irregulares del aneurisma.

- El aneurisma más proximal.

\section{Complicaciones HSA}

Las principales complicaciones y su tratamiento más adecuado tras la HSA son:

\section{Resangrado}

Varios estudios prospectivos han mostrado un índice de sangrado similar ${ }^{108,111,160,225}$. Tras una HSA existe un riesgo de resangrado de un $3-4 \%$ en las primeras 24 horas, seguido de un riesgo acumulativo entre un 1-2\%/día durante las primeras cuatro semanas. En los casos que se trataron conservadoramente, las incidencias de resangrado registradas en el primer mes oscilaron entre 20-30\%, estabilizándose después del tercer año. En el Estudio Cooperativo $^{160}$ con un mayor seguimiento, encontraron un índice de resangrado del 2.2\%/año pasados 6 meses de la hemorragia durante los primeros 10 años, descendiendo a un $0.86 \%$ /año a partir de la segunda década. La mortalidad ligada al resangrado se cifra en un $74 \%{ }^{108}$.El tratamiento fundamental para prevenir el resangrado es la oclusión del aneurisma (quirúrgica/embolización). El uso de fibrinolíticos en dosis altas (ácido épsilon-aminocaproico $1 \mathrm{~g} / 6$ horas i.v.) durante las primeras 72 horas del sangrado reduce el riesgo de resangrado sin que se produzca un incremento en complicaciones isquémicas ${ }^{202}$. Esta estrategia podría ser útil en los casos en los que fuera necesario llevar a cabo un traslado de centro hospitalario para el tratamiento del aneurisma.

\section{Vasoespasmo/isquemia}

El vasoespasmo es una respuesta arterial a los depósitos de sangre subaracnoidea, probablemente secundaria a sustancias liberadas por la lisis del coágulo; los pacientes con gruesos coágulos cisternales están expuestos a una mayor incidencia de déficits isquémicos ${ }^{61}$. El vasoespasmo angiográfico tiene un pico de incidencia alrededor del $7^{\circ}$ día, con una resolución gradual entre 2 y 4 semanas. La incidencia estimada de vasoespasmo angiográfico es de aproximadamente $2 / 3$ de los pacientes, de los cuales $1 / 3$ desarrollará síntomas isquémicos. El vasoespasmo era el motivo de fallecimiento en el $40 \%$ de los pacientes en los años 60 , pero esta cifra ha descendido a un $7 \%$ en la actualidad $^{89,109 .}$

El desarrollo de un nuevo déficit focal, no explicable por la hidrocefalia o el resangrado, es el primer síntoma objetivo del vasoespasmo. Cada vez es más evidente que el vasoespasmo puede aparecer sin síntomas evidentes en pacientes comatosos y ser causa de la aparición de infartos cerebrales. Por ello la sospecha debe ser mayor en pacientes con mal grado clínico que por otro lado tienen más riesgo de presentar vasoespasmo.

Existen métodos de monitorización de la presencia de vasoespasmo como es el doppler transcraneal (DTC). Este método depende del observador que la realiza y requiere del establecimiento de valores umbrales específicos para cada centro. Los valores absolutos pueden ser engañosos pero parece que el índice de Lindegaard puede ser útil en la detección del vasoespasmo ${ }^{79,106,133,140,154,156}$. Es necesario realizar una monitorización a lo largo de los días de la evolución de la HSA dado que los cambios en las mediciones, tales como aceleración del registro o incremento de la pulsatilidad también pueden predecir el vasoespasmo. La TC de perfusión puede ser un método útil, sin embargo, recientemente se han observado efectos secundarios debidos a la excesiva radiación a los que se somete a los enfermos si se repiten con frecuencia las exploraciones.

El mejor tratamiento de la isquemia secundaria a vasoespasmo es la prevención del mismo. Para ello, fundamentalmente, existen varias medidas:

1. Normovolemia. La volemia puede ser un factor influyente importante entre pacientes con vasoespasmo cerebral asintomático y sintomático. Los pacientes con volemia baja y vasoespasmo es más probable que desarrollen eventos isquémicos diferidos, en comparación con los pacientes con volumen sanguíneo normal, que tienen menos probabilidad de desarrollar isquemia cerebral aunque tengan vasoespasmo. Sin embargo, el tratamiento con hipervolemia tras clipaje del aneurisma en la HSA 
no incrementa el flujo sanguíneo cerebral ni el volumen sanguíneo, comparado con la normovolemia. La administración de fluidos es importante para evitar la hipovolemia, pero la hipervolemia profiláctica no confiere ningún beneficio. La recomendación de triple $\mathrm{H}$ para la prevención y tratamiento del vasoespasmo y de las complicaciones isquémicas, tiene un nivel de evidencia III de V, grado C.

En conclusión, en la HSA aneurismática, se propone mantener a los pacientes normovolémicos, sin que haya evidencia en la actualidad de que el intento de mantener situaciones de hipervolemia prevenga la aparición de vasoespasmo o complicaciones isquémicas. Se propone que en los grados Hunt-Hess entre 3 y 5 y/o Fisher superior o igual a 3 , la volemia sea monitorizada mediante presión venosa central (PVC).

2. Antagonistas del calcio: nimodipino. Hay bastantes estudios que demuestran que el nimodipino reduce la incidencia déficits neurológicos isquémicos y mejora la evolución neurológica a los 3 meses. Se recomienda nimodipino oral, para la reducción de complicaciones asociadas al vasoespasmo cerebral.

El tratamiento con nimodipino oral (60 mg cada cuatro horas, continuado por tres semanas) es el tratamiento de elección en pacientes con una hemorragia subaracnoidea aneurismática. Recientemente se ha comprobado la similar efectividad del nimodipino intravenoso aunque es de destacar el riesgo de hipotensión ${ }^{121}$.

En cuanto al tratamiento del vasoespasmo éste radica en dos posible medidas que son el tratamiento mediante triple $\mathrm{H}$ o mediante métodos endovasculares.

La terapia Triple- $H^{13,167,179}$ (Hipertensión: TAS $>150$ mm Hg. Hemodilución: hematocrito alrededor del 30\% e Hipervolemia: presión venosa central 5-10 mm H2O) se recomienda para disminuir las complicaciones producidas por el vasoespasmo. No existen estudios randomizados que demuestren su beneficio, ni tampoco se conoce cuál de las tres opciones es la más beneficiosa ${ }^{209}$. La hipervolemiahemodilución aumenta el volumen sanguíneo y el gasto cardíaco y mejora teóricamente las propiedades reológicas de la microcirculación cerebral a nivel de la penumbra isquémica por disminución de la viscosidad sanguínea. El aumento del gasto cardíaco no afecta al FSC de las zonas no isquémicas del cerebro. Las zonas isquémicas tienen un FSC que es presión dependiente por pérdida de la autorregulación. El inconveniente de este tratamiento es el aumento del FSC en las áreas cerebrales que tienen alterada la barrera hematoencefálica, que no sólo puede aumentar el edema cerebral sino también agravar la isquemia por la reperfusión ${ }^{95}$. En cuanto a la hipertensión hay que tener en cuenta que hasta el 34\% del total de los pacientes desarrollan vasoespasmo sintomático a pesar de la terapia hipervolémica por lo que hay que recurrir a la terapia hiperdinámica para tratar de revertir los sínto- mas del vasoespasmo. Se recomienda utilizarla sólo con el aneurisma clipado o excluido de la circulación tras embolización. Puede tener complicaciones sistémicas como edema pulmonar o isquemia miocárdica especialmente con el uso de elevadas dosis de vasopresores. Se han utilizado varias aminas vasoactivas para conseguir inducir HTA, entre ellas noradrenalina, dobutamina (DBT) y fenilefrina. Todos ellos pueden tener efectos adversos potenciales. Estos pacientes tienen que estar estrechamente vigilados en UCI ya que pueden tener múltiples complicaciones neurológicas y sistémicas.

El uso de otras sustancias como Tirilazad, magnesio, corticoides, etc, todavía se mantiene en fase de investigación ${ }^{186}$.

En cuanto a los métodos endovasculares para el tratamiento del vasoespasmo se emplean dos tipos:

1. La infusión intraarterial de fármacos vasodilatadores.

2. La angioplastia con balón.

1. El tratamiento farmacológico intraarterial tiene su origen en el uso de los vasodilatadores alcaloides (papaverina) intraoperatorios. Se han usado tanto alcaloides como antagonistas del calcio. La papaverina es un alcaloide con una vida media de alrededor de 2 horas. La técnica consiste en la infusión intraarterial de una dosis de 100 a $300 \mathrm{mg}$ de papaverina en $100 \mathrm{ml}$, por territorio en unos 60 minutos $(3 \mathrm{mg} / \mathrm{ml} \text { a } 6-9 \mathrm{ml} / \mathrm{min})^{42}$. Como la absorción es trans-endotelial, los mejores resultados se obtienen cuando la infusión es próxima a la zona de vasoespasmo. Aunque en el $80-95 \%$ de casos se observa una respuesta angiográfica, sólo un 25$50 \%$ de pacientes presentan una respuesta clínica ${ }^{135}$. La administración de papaverina intraarterial tiene una eficacia limitada supeditada a su efecto temporal (no superior a 24-48h) lo cual puede obligar a nueva infusión y una serie de desventajas ligadas a sus efectos colaterales, tales como reducción de la presión arterial, taquicardia, aumento de la presión intracraneal, depresión respiratoria ${ }^{138}$. Los efectos secundarios y la corta vida media de la papaverina han hecho que se hayan ensayado otros fármacos intrarteriales ${ }^{52,120}$. Uno de ellos es el verapamilo, ampliamente usado para tratar el vasoespasmo coronario. Su vida media es de 7 horas y aumenta el flujo sanguíneo cerebral sin aumentar la PIC ni la presión arterial. Otro fármaco utilizado intrarterialmente es el nicardipino, de vida media en torno a 16 horas. Su uso intrarterial evita los efectos secundarios de la administración intravenosa (hipotensión, edema pulmonar, insuficiencia renal) aunque se han descrito casos de aumento de la PIC. El nimodipino, otro 
antagonista del calcio, también se está utilizando intrarterialmente. Su vida media es alrededor de 9 horas y durante su uso no se han descrito complicaciones, por lo que su uso se está extendiendo. Otros fármacos como el fasudil, la milrinona y el colforsin han sido también utilizados de forma intrarterial para el tratamiento del vasoespasmo de forma puntual en algunos estudios publicados con resultados favorables. Recientemente se ha introducido el uso de la nicardipina intrarterial como vasodilatador ${ }^{192}$.

2. La angioplastia con balón fue introducida por Zubkov en 1984 para el tratamiento del vasoespasmo grave, con resultados más efectivos y duraderos que la angioplastia farmacológica, pero con un incremento del riesgo de complicaciones ${ }^{231}$. Debido a su potencial riesgo de complicaciones graves debe restringirse la angioplastia con balón a aquellos pacientes con vasoespasmo sintomático y con fallo de los demás tratamientos menos invasivos ${ }^{148}$. El intervalo de tiempo para realizar la angioplastia es controvertido, se discute entre 6 y $12 \mathrm{~h}$, pero se debería de aplicar antes que la isquemia progrese a infarto ${ }^{53}$. La plasticidad del vaso afecto de vasoespasmo disminuye con el paso del tiempo y aumenta la presión que se debe ejercer con el balón de angioplastia, aumentando el riesgo de ruptura de la pared arterial. Por todo ello, la indicación de la angioplastia debe ser considerada de forma individual y ser analizado junto con la clínica neurológica, el DTC y el estudio angiográfico que, además del vasoespasmo muestra una zona de hipoperfusión cerebral distal.

El tratamiento endovascular del paciente cuyo vasoespasmo se convierte en sintomático debe ser propuesto según los siguientes criterios ${ }^{10,31}$ :

- Aparición de un déficit no atribuible a otra causa.

- Déficit refractario al aumento de tensión arterial.

- Ausencia de infarto visible en la TC.

- Vasoespasmo angiográficamente evidente en una localización que podría explicar el déficit con hipoperfusión distal de este territorio vascular.

Se podría plantear la siguiente propuesta de cuidados según la sospecha clínica o los síntomas de isquemia:

- Sintomas leves de isquemia

- Se realizará TC craneal y analítica para descartar otras causas y se intentará confirmar y establecer el grado de isquemia por vasoespasmo cerebral mediante DTC.

- Evitar la hipovolemia (3000 cc de cristaloides/24h) control de PVC (entre 5-10 cm de H2O).

- Modificar las características reológicas (coloides, hematocrito entre 30-35\%, manitol, albúmina).

- Evitar la hipertensión arterial si el aneurisma no está clipado o embolizado.
- En las situaciones clínicas de WFNS grados I-II-III valorar la asociación de patología cardiovascular previa o edades avanzadas de los pacientes por el riesgo de complicaciones asociadas al tratamiento: EAP, IAM, hiponatremia dilucional. Estos pacientes deben ingresar en una Unidad de Críticos para una mejor monitorización y control hemodinámico.

- Síntomas graves de isquemia

- Comenzar las maniobras previas y trasladar al paciente a una Unidad de Críticos para intensificar el tratamiento triple $\mathrm{H}$ con fármacos vasopresores sistémicos.

- Valorar arteriografía cerebral con angioplastia y/o nimodipino intrarterial si no remite la sintomatología atribuible al vasoespasmo, a pesar de medidas máximas, especialmente la hipertensión arterial.

\section{Hidrocefalia}

Una dilatación ventricular (Indice de Evans: distancia entre cuernos frontales/diámetro entre ambas tablas internas al mismo nivel $>0.30$ ) puede aparecer en el periodo agudo entre un $20-40 \%$ de los $\operatorname{casos}^{78,87}$. Si el grado clínico es aceptable (I-III) y no existe deterioro neurológico, se puede adoptar una postura expectante ya que en la mayoría de los casos ocurre una resolución espontánea. Si existe deterioro clínico, se aconseja un drenaje ventricular externo, asumiéndose un aumento del riesgo de meningitis y de resangrado. Si el paciente se encuentra en grados IV-V se recomienda drenaje externo ${ }^{119,181}$.

A pesar de que en algunos estudios el uso de drenajes ventriculares en estos pacientes se ha asociado a un incremento del riesgo de resangrado, no hay una evidencia firme en este sentido que contraindique su uso ${ }^{65}$. El uso de drenajes externos se ha asociado a infección del líquido cefalorraquídeo ${ }^{28}$, mayor cuanto mayor sea la hemorragia subaracnoidea o intraventricular y la duración del drenaje. En este sentido no parecer útil el uso de antibioterapia profiláctica tras su implantación ${ }^{8}$. La introducción de catéteres impregnados en antibióticos parece útil en la prevención de la infección asociada a drenajes ventriculares en pacientes con hemorragia intraventricular ${ }^{198,229}$. Por otro lado se ha intentado tratar la hemorragia intraventricular con fibrinolíticos instilados a través del drenaje ventricular ${ }^{69,158,208}$. Con esta maniobra se pretende disminuir el efecto nocivo de la sangre intraventricular sobre la evolución final y disminuir el riesgo de vasoespasmo y daño isquémico ${ }^{132}$. No obstante parece que el efecto que tiene esta medida es mejorar el manejo de los pacientes portadores de drenajes ya que disminuye su tasa de obstrucción y por lo tanto la necesidad de su recambio ${ }^{152,214}$.

La hidrocefalia crónica acompañada de síntomas clínicos compatibles con una hidrocefalia normotensa se debe tratar con una derivación ventrículo peritoneal. Aparece en 
torno a un 18 a $26 \%$ de los supervivientes. Los factores que favorecen su aparición son la edad, la presencia de hidrocefalia al ingreso, el sangrado intraventricular y su cuantía y un mal grado clínico.

\section{Convulsiones}

Aunque no existe una fuerte evidencia científica, algunos autores recomiendan la administración de anticonvulsivos con el fin de disminuir el riesgo de resangrado tras una crisis. Sin embargo, otros autores afirman que deben usarse sólo en el caso de pacientes con riesgo de presentar convulsiones (por patología previa, aneurismas de arteria cerebral media, infartos cerebrales) dado que su uso puede conllevar especialmente en el caso de la fenitoína peor recuperación o deterioro cognitivo ${ }^{230}$. El levetiracetam podría ser superior a la fenitoina debido a su mayor tolerabilidad y ausencia de asociación a peor pronóstico neurológico ${ }^{196,204}$. El uso a largo plazo no está recomendado en pacientes libres de convulsiones, excepto en pacientes con antecedentes de convulsiones, hematomas intraparenquimatosos o infartos. Se puede pues considerar su aplicación temporal en el periodo posthemorrágico inmediato pero su uso a más largo plazo no está recomendado.

\section{Bibliografía}

1. Report of World Federation of Neurological Surgeons Committee on a Universal Subarachnoid Hemorrhage Grading Scale. J Neurosurg 1988; 68: 985-986.

2. Unruptured intracranial aneurysms--risk of rupture and risks of surgical intervention. International Study of Unruptured Intracranial Aneurysms Investigators. N Engl J Med 1998; 339: 1725-1733.

3. Risks and benefits of screening for intracranial aneurysms in first-degree relatives of patients with sporadic subarachnoid hemorrhage. N Engl J Med 1999; 341: 1344-1350.

4. Epidemiology of aneurysmal subarachnoid hemorrhage in Australia and New Zealand: incidence and case fatality from the Australasian Cooperative Research on Subarachnoid Hemorrhage Study (ACROSS). Stroke 2000; 31: 1843-1850.

5. Abe, M., Tabuchi, K., Yokoyama, H., Uchino, A.: Blood blisterlike aneurysms of the internal carotid artery. J Neurosurg 1998; 89: 419-424.

6. Alberts, M.J.: Genetics of cerebrovascular disease. Stroke 2004; 35: 342-344.

7. Alberts, M.J., Quinones, A., Graffagnino, C., Friedman, A., Roses, A.D.: Risk of intracranial aneurysms in families with subarachnoid hemorrhage. Can J Neurol Sci 1995; 22: 121-125.

8. Alleyne, C.H., Jr., Hassan, M., Zabramski, J.M.: The efficacy and cost of prophylactic and perioprocedural antibiotics in patients with external ventricular drains. Neurosurgery
2000; 47: 1124-1127.

9. Alvord, E.C., Jr. Thorn, R.B.: Natural history of subarachnoid hemorrhage: early prognosis. Clin Neurosurg 1977; 24: 167-175.

10. Andaluz, N., Tomsick, T.A., Tew, J.M., Jr., van Loveren, H.R., Yeh, H.S., Zuccarello, M.: Indications for endovascular therapy for refractory vasospasm after aneurysmal subarachnoid hemorrhage: experience at the University of Cincinnati. Surg Neurol 2002; 58: 131-138.

11. Anderson, G.B., Steinke, D.E., Petruk, K.C., Ashforth, R., Findlay, J.M.: Computed tomographic angiography versus digital subtraction angiography for the diagnosis and early treatment of ruptured intracranial aneurysms. Neurosurgery 1999; 45: 1315-1320.

12. Ausman, J.I. Roitberg, B.: A response from the ISUIA. International Study on Unruptured Intracranial Aneurysms. Surg Neurol 1999; 52: 428-430.

13. Awad, I.A., Carter, L.P., Spetzler, R.F., Medina, M., Williams, F.C., Jr.: Clinical vasospasm after subarachnoid hemorrhage: response to hypervolemic hemodilution and arterial hypertension. Stroke 1987; 18: 365-372.

14. Bailes, J.E., Spetzler, R.F., Hadley, M.N., Baldwin, H.Z.: Management morbidity and mortality of poor-grade aneurysm patients. J Neurosurg 1990; 72: 559-566.

15. Bakshi, R., Kamran, S., Kinkel, P.R., Bates, V.E., Mechtler, L.L., Janardhan, V., Belani, S.L., Kinkel, W.R.: Fluid-attenuated inversion-recovery MR imaging in acute and subacute cerebral intraventricular hemorrhage. AJNR Am J Neuroradiol 1999; 20: 629-636.

16. Bano-Ruiz, E., Abarca-Olivas, J., Duart-Clemente, J.M., Ballenilla-Marco, F., García, P., Botella-Asuncion, C.: Influencia de los cambios de presión atmosférica y otras variantes meteorológicas en la incidencia de la hemorragia subaracnoidea. Neurocirugia 2010; 21: 14-21.

17. Barker, F.G., Amin-Hanjani, S., Butler, W.E., Ogilvy, C.S., Carter, B.S.: In-hospital mortality and morbidity after surgical treatment of unruptured intracranial aneurysms in the United States, 1996-2000: the effect of hospital and surgeon volume. Neurosurgery 2003; 52: 995-1007.

18. Barker, F.G. Ogilvy, C.S.: Efficacy of prophylactic nimodipine for delayed ischemic deficit after subarachnoid hemorrhage: a metaanalysis. J Neurosurg 1996; 84: 405-414.

19. Baskaya, M.K., Ahmed, A.S., Ates, O., Niemann, D.: Surgical treatment of blood blister-like aneurysms of the supraclinoid internal carotid artery with extracranial-intracranial bypass and trapping. Neurosurg Focus 2008; 24: E13.

20. Bassi, P., Bandera, R., Loiero, M., Tognoni, G., Mangoni, A.: Warning signs in subarachnoid hemorrhage: a cooperative study. Acta Neurol Scand 1991; 84: 277-281.

21. Bederson, J.B., Awad, I.A., Wiebers, D.O., Piepgras, D., Haley, E.C., Jr., Brott, T., Hademenos, G., Chyatte, D., Rosenwasser, R., Caroselli, C.: Recommendations for the management of patients with unruptured intracranial aneu- 
rysms: A statement for healthcare professionals from the Stroke Council of the American Heart Association. Circulation 2000; 102: 2300-2308.

22. Bederson, J.B., Connolly, E.S., Jr., Batjer, H.H., Dacey, R.G., Dion, J.E., Diringer, M.N., Duldner, J.E., Jr., Harbaugh, R.E., Patel, A.B., Rosenwasser, R.H.: Guidelines for the management of aneurysmal subarachnoid hemorrhage: a statement for healthcare professionals from a special writing group of the Stroke Council, American Heart Association. Stroke 2009; 40: 994-1025.

23. Bederson, J.B., Connolly, E.S., Jr., Batjer, H.H., Dacey, R.G., Dion, J.E., Diringer, M.N., Duldner, J.E., Jr., Harbaugh, R.E., Patel, A.B., Rosenwasser, R.H.: Guidelines for the management of aneurysmal subarachnoid hemorrhage: a statement for healthcare professionals from a special writing group of the Stroke Council, American Heart Association. Stroke 2009; 40: 994-1025.

24. Bergdal, O., Springborg, J., Hauerberg, J., Eskesen, V., Poulsgaard, L., Romner, B.: Outcome after emergency surgery without angiography in patients with intracerebral haemorrhage after aneurysm rupture. Acta Neurochir (Wien) 2009; 151: 911-915.

25. Berman, M.F., Solomon, R.A., Mayer, S.A., Johnston, S.C., Yung, P.P.: Impact of hospital-related factors on outcome after treatment of cerebral aneurysms. Stroke 2003; 34: 22002207.

26. Boet, R., Poon, W.S., Lam, J.M., Yu, S.C.: The surgical treatment of intracranial aneurysms based on computer tomographic angiography alone--streamlining the acute mananagement of symptomatic aneurysms. Acta Neurochir (Wien) 2003; 145: 101-105.

27. Bossuyt, P.M., Raaymakers, T.W., Bonsel, G.J., Rinkel, G.J.: Screening families for intracranial aneurysms: anxiety, perceived risk, and informed choice. Prev Med 2005; 41: 795-799.

28. Bota, D.P., Lefranc, F., Vilallobos, H.R., Brimioulle, S., Vincent, J.L.: Ventriculostomy-related infections in critically ill patients: a 6-year experience. J Neurosurg 2005; 103: 468-472.

29. Bracard, S., Abdel-Kerim, A., Thuillier, L., Klein, O., Anxionnat, R., Finitsis, S., Lebedinsky, A., de Freitas, C.M., Pinheiro, N., de Andrade, G.C., Picard, L.: Endovascular coil occlusion of 152 middle cerebral artery aneurysms: initial and midterm angiographic and clinical results. J Neurosurg 2010; 112: 703-708.

30. Brilstra, E.H., Rinkel, G.J., van der G.Y., van Rooij, W.J., Algra, A.: Treatment of intracranial aneurysms by embolization with coils: a systematic review. Stroke 1999; 30: 470476.

31. Brisman, J.L., Eskridge, J.M., Newell, D.W.: Neurointerventional treatment of vasospasm. Neurol Res 2006; 28 : 769-776.

32. Bromberg, J.E., Rinkel, G.J., Algra, A., Greebe, P., van Duyn, C.M., Hasan, D., Limburg, M., ter Berg, H.W., Wijdicks, E.F., van Gijn, J.: Subarachnoid haemorrhage in first and second degree relatives of patients with subarachnoid haemorrhage. BMJ 1995; 311: 288-289.

33. Brown, R.D., Jr., Huston, J., Hornung, R., Foroud, T., Kallmes, D.F., Kleindorfer, D., Meissner, I., Woo, D., Sauerbeck, L., Broderick, J.: Screening for brain aneurysm in the Familial Intracranial Aneurysm study: frequency and predictors of lesion detection. J Neurosurg 2008; 108: 1132-1138.

34. Butler, W.E., Barker, F.G., Crowell, R.M.: Patients with polycystic kidney disease would benefit from routine magnetic resonance angiographic screening for intracerebral aneurysms: a decision analysis. Neurosurgery 1996; 38: 506515.

35. Buxton, N., Liu, C., Dasic, D., Moody, P., Hope, D.T.: Relationship of aneurysmal subarachnoid hemorrhage to changes in atmospheric pressure: results of a prospective study. J Neurosurg 2001; 95: 391-392.

36. Byrne, J.V., Sohn, M.J., Molyneux, A.J., Chir, B.: Fiveyear experience in using coil embolization for ruptured intracranial aneurysms: outcomes and incidence of late rebleeding. J Neurosurg 1999; 90: 656-663.

37. Caicoya, M., Rodriguez, T., Lasheras, C., Cuello, R., Corrales, C., Blazquez, B.: [Stroke incidence in Asturias, 1990-1991]. Rev Neurol 1996; 24: 806-811.

38. Cesarini, K.G., Hardemark, H.G., Persson, L: Improved survival after aneurysmal subarachnoid hemorrhage: review of case management during a 12-year period. J Neurosurg 1999; 90: 664-672.

39. Choi, D.S., Kim, M.C., Lee, S.K., Willinsky, R.A., terBrugge, K.G.: Clinical and angiographic long-term follow-up of completely coiled intracranial aneurysms using endovascular technique. J Neurosurg 2010; 112: 575-581.

40. Chwajol, M., Starke, R.M., Kim, G.H., Mayer, S.A., Connolly, E.S.: Antifibrinolytic therapy to prevent early rebleeding after subarachnoid hemorrhage. Neurocrit Care 2008; 8: 418-426.

41. Claassen, J., Bernardini, G.L., Kreiter, K., Bates, J., Du, Y.E., Copeland, D., Connolly, E.S., Mayer, S.A.: Effect of cisternal and ventricular blood on risk of delayed cerebral ischemia after subarachnoid hemorrhage: the Fisher scale revisited. Stroke 2001; 32: 2012-2020.

42. Clouston, J.E., Numaguchi, Y., Zoarski, G.H., Aldrich, E.F., Simard, J.M., Zitnay, K.M.: Intraarterial papaverine infusion for cerebral vasospasm after subarachnoid hemorrhage. AJNR Am J Neuroradiol 1995; 16: 27-38.

43. Cross, D.T., III, Tirschwell, D.L., Clark, M.A., Tuden, D., Derdeyn, C.P., Moran, C.J., Dacey, R.G., Jr.: Mortality rates after subarachnoid hemorrhage: variations according to hospital case volume in 18 states. J Neurosurg 2003; 99: 810817.

44. Dammert, S., Krings, T., Moller-Hartmann, W., Ueffing, E., Hans, F.J., Willmes, K., Mull, M., Thron, A.: 
Detection of intracranial aneurysms with multislice CT: comparison with conventional angiography. Neuroradiology 2004; 46: 427-434.

45. de Gans, K., Nieuwkamp, D.J., Rinkel, G.J., Algra, A.: Timing of aneurysm surgery in subarachnoid hemorrhage: a systematic review of the literature. Neurosurgery 2002; 50: 336-340.

46. de Rooij, N.K., Linn, F.H., van der Plas, J.A., Algra, A., Rinkel, G.J.: Incidence of subarachnoid haemorrhage: a systematic review with emphasis on region, age, gender and time trends. J Neurol Neurosurg Psychiatry 2007; 78: 13651372.

47. de Toledo, P., Rios, P.M., Ledezma, A., Sanchís, A., Alén, J.F., Lagares, A.: Predicting the outcome of patients with subarachnoid hemorrhage using machine learning techniques. IEEE Trans Inf Technol Biomed 2009; 13: 794-801.

48. Dehdashti, A.R., Rufenacht, D.A., Delavelle, J., Reverdin, A., de Tribolet, N..: Therapeutic decision and management of aneurysmal subarachnoid haemorrhage based on computed tomographic angiography. Br J Neurosurg 2003;17: 46-53.

49. Díez Lobato, R., Porto, J., Gómez, P.A., Campollo, J., Alday, R., González, P., Rodríguez Boto, G., de la Lama, A.: Aneurisma en ampolla de la carótida asociado a aneurismas múltiples y malformación arteriovenosa cerebral tratado con oclusión carotídea y bypass de alto flujo. Neurocirugía 2000; 11: 435-439.

50. Disney, L., Weir, B., Grace, M.: Factors influencing the outcome of aneurysm rupture in poor grade patients: a prospective series. Neurosurgery 1988; 23: 1-9.

51. Dorsch, N.W., Besser, M., Brazenor, G.A., Stuart, G.G.: Timing of surgery for cerebral aneurysms: a plea for early referral. Med J Aust 1989; 150: 183, 187-183, 188.

52. Eddleman, C.S., Hurley, M.C., Naidech, A.M., Batjer, H.H., Bendok, B.R.: Endovascular options in the treatment of delayed ischemic neurological deficits due to cerebral vasospasm. Neurosurg Focus 2009; 26: E6.

53. Eskridge, J.M., McAuliffe, W., Song, J.K., Deliganis, A.V., Newell, D.W., Lewis, D.H., Mayberg, M.R., Winn, H.R.: Balloon angioplasty for the treatment of vasospasm: results of first 50 cases. Neurosurgery 1998; 42: 510-516.

54. Farb, R.I., Nag, S., Scott, J.N., Willinsky, .R.A, Marotta, T.R., Montanera, W.J., Tomlinson, G., terBrugge, K.G.: Surveillance of intracranial aneurysms treated with detachable coils: a comparison of MRA techniques. Neuroradiology 2005; 47: 507-515.

55. Fehlings, M,G., Gentili, F.: The association between polycystic kidney disease and cerebral aneurysms. Can J Neurol Sci 1991; 18: 505-509.

56. Feigin, V.L., Rinkel, G.J., Algra, A., Vermeulen, M., van Gijn, J.: Calcium antagonists in patients with aneurysmal subarachnoid hemorrhage: a systematic review. Neurology 1998; 50: 876-883.
57. Feigin, V.L., Rinkel, G.J., Lawes, C.M., Algra, A., Bennett, D.A., van Gijn, J., Anderson, C.S.: Risk factors for subarachnoid hemorrhage: an updated systematic review of epidemiological studies. Stroke 2005; 36: 2773-2780.

58. Ferns, S.P., Sprengers, M.E., van Rooij, W.J., Rinkel, G.J., van Rijn, J.C., Bipat, S., Sluzewski, M., Majoie, C.B.: Coiling of intracranial aneurysms: a systematic review on initial occlusion and reopening and retreatment rates. Stroke 2009; 40: e523-e529.

59. Fiebach, J.B., Schellinger, P.D., Geletneky, K., Wilde, P., Meyer, M., Hacke, W., Sartor, K.: MRI in acute subarachnoid haemorrhage; findings with a standardised stroke protocol. Neuroradiology 2004; 46: 44-48.

60. Findlay, J.M.: Current management of aneurysmal subarachnoid hemorrhage guidelines from the Canadian Neurosurgical Society. Can J Neurol Sci 1997; 24: 161-170.

61. Fisher, C.M., Kistler, J.P., Davis, J.M.: Relation of cerebral vasospasm to subarachnoid hemorrhage visualized by computerized tomographic scanning. Neurosurgery 1980; 6: $1-9$.

62. Fodstad, H.: Antifibrinolytic treatment in subarachnoid haemorrhage: present state. Acta Neurochir (Wien) 1982; 63: 233-244.

63. Fogelholm, R.: Subarachnoid hemorrhage in middleFinland: incidence, early prognosis and indications for neurosurgical treatment. Stroke 1981; 12: 296-301.

64. Fogelholm, R., Hernesniemi, J., Vapalahti, M..: Impact of early surgery on outcome after aneurysmal subarachnoid hemorrhage. A population-based study. Stroke 1993; 24:1 649-1654.

65. Fountas, K.N., Kapsalaki, E.Z., Machinis, T., Karampelas, I., Smisson, H.F., Robinson, J.S.: Review of the literature regarding the relationship of rebleeding and external ventricular drainage in patients with subarachnoid hemorrhage of aneurysmal origin. Neurosurg Rev 2006 ;29: 14-18.

66. Gaist, D., Vaeth, M., Tsiropoulos, I., Christensen, K., Corder, E., Olsen, J., Sorensen, H.T.: Risk of subarachnoid haemorrhage in first degree relatives of patients with subarachnoid haemorrhage: follow up study based on national registries in Denmark. BMJ 2000; 320: 141-145.

67. García Allut, A., Bollar, A., Prieto, A., Gelabert, M. Martínez Rumbo, R.: Aneurismas múltiples. Problemática y actitudes. Neurocirugía 1993; 4: 269-274.

68. Giannotta, S.L. Kindt, G.W.: Total morbidity and mortality rates of patients with surgically treated intracranial aneurysms. Neurosurgery 1979; 4: 125-128.

69. Goh, K.Y., Hsiang, J.N., Zhu, X.L., Poon, W.S.: Intraventricular recombinant tissue plasminogen activator for treatment of spontaneous intraventricular haemorrhage in pregnancy. J Clin Neurosci 1999; 6: 158-159.

70. Gomez, P.A., Lobato, R.D., Rivas, J.J., Cabrera, A., Sarabia, R., Castro, S., Castaneda, M., Canizal, J.M.: Subarachnoid haemorrhage of unknown aetiology. Acta Neurochir 
(Wien) 1989; 101: 35-41

71. González-Darder, J.M. y Gil-Salú, J.L.: La técnica de Dallas en el tratamiento de los aneurismas de la arteria oftálmica de gran tamaño. Neurocirugía 1999; 10: 55-60.

72. González-Darder, J.M., Cervera, J., Gómez, R., Pesudo, J.V., Tudela, J., García-Vila, J.: Experiencia inicial con la angio-TC-3D en el manejo de la hemorragia subaracnoidea y de los aneurismas cerebrales. Neurocirugía 2000; 11: 23-30.

73. Gonzalez-Darder, J.M., Pesudo-Martinez, J.V., FeliuTatay, R.A.: Microsurgical management of cerebral aneurysms based in CT angiography with three-dimensional reconstruction (3D-CTA) and without preoperative cerebral angiography. Acta Neurochir (Wien) 2001; 143: 673-679.

74. González-Darder, J.M., Feliú, R., Pesudo, J.V., Borrás, J.M., Gómez, R., Díaz, C., Lázaro, S. García Vila, J.: Tratamiento quirúrgico de los aneurismas de la arteria comunicante anterior basado en el estudio de angio-TAC con reconstrucción tridimensional y sin angiografia preoperatoria. Neurocirugía 2002; 13: 446-454.

75. González-Darder, J.M., Feliú, R., Pesudo, J.V., Borrás, J.M., Gómez, R., Díaz, C., Lázaro, S., Redondo, M. García Vila, J.: Tratamiento quirúrgico de los aneurismas de la arteria comunicante posterior basado en el estudio de angio-TAC con reconstrucción tridimensional y sin angiografia preoperatoria. Neurocirugía 2003; 14: 207-215.

76. González-Pérez, M.I.: Resultado del tratamiento de la hemorragia subaracnoidea debida a rotura de aneurismas cerebrales. Neurocirugía 2006;17: 433-439.

77. Gotoh, O., Tamura, A., Yasui, N., Suzuki, A., Hadeishi, H., Sano, K.: Glasgow Coma Scale in the prediction of outcome after early aneurysm surgery. Neurosurgery 1996; 39: 19-24.

78. Graff-Radford, N.R., Torner, J., Adams, H.P., Jr., Kassell, N.F.: Factors associated with hydrocephalus after subarachnoid hemorrhage. A report of the Cooperative Aneurysm Study. Arch Neurol 1989; 46: 744-752.

79. Grosset, D.G., Straiton, J., Du, T.M., Bullock, R.: Prediction of symptomatic vasospasm after subarachnoid hemorrhage by rapidly increasing transcranial Doppler velocity and cerebral blood flow changes. Stroke 1992; 23: 674-679.

80. Gruber, D.P., Zimmerman, G.A., Tomsick, T.A., van Loveren, H.R., Link, M.J., Tew, J.M., Jr.: A comparison between endovascular and surgical management of basilar artery apex aneurysms. J Neurosurg 1999; 90: 868-874.

81. Guglielmi, G,. Vinuela, F.: Intracranial aneurysms. Guglielmi electrothrombotic coils. Neurosurg Clin N Am 1994; 5: 427-435.

82. Guglielmi, G., Vinuela, F., Dion, J., Duckwiler, G.: Electrothrombosis of saccular aneurysms via endovascular approach. Part 2: Preliminary clinical experience. J Neurosurg 1991; 75: 8-14.

83. Guglielmi, G., Vinuela, F., Duckwiler, G., Dion, J., Lylyk, P., Berenstein, A., Strother, C., Graves, V., Halbach, V.,
Nichols, D.: Endovascular treatment of posterior circulation aneurysms by electrothrombosis using electrically detachable coils. J Neurosurg 1992; 77: 515-524.

84. Hadeishi, H., Suzuki, A., Yasui, N., Hatazawa, J., Shimosegawa, E.: Diffusion-weighted magnetic resonance imaging in patients with subarachnoid hemorrhage. Neurosurgery 2002; 50: 741-747.

85. Haley, E.C., Jr., Kassell, N.F., Torner, J.C.: The International Cooperative Study on the Timing of Aneurysm Surgery. The North American experience. Stroke 1992; 23: 205-214.

86. Hamada, J., Morioka, M., Yano, S., Kai, Y., Ushio, Y.: Incidence and early prognosis of aneurysmal subarachnoid hemorrhage in Kumamoto Prefecture, Japan. Neurosurgery 2004; 54: 31-37.

87. Hasan, D., Vermeulen, M., Wijdicks, E.F., Hijdra, A., van Gijn, J.: Management problems in acute hydrocephalus after subarachnoid hemorrhage. Stroke 1989; 20: 747-753.

88. Heiskanen, O., Poranen, A., Kuurne, T., Valtonen, S., Kaste, M.: Acute surgery for intracerebral haematomas caused by rupture of an intracranial arterial aneurysm. A prospective randomized study. Acta Neurochir (Wien) 1988; 90: 81-83.

89. Hijdra, A., Braakman, R., van Gijn, J., Vermeulen, M., van Crevel, H.: Aneurysmal subarachnoid hemorrhage. Complications and outcome in a hospital population. Stroke 1987; 18: 1061-1067.

90. Hillman, J., Fridriksson, S., Nilsson, O., Yu, Z., Saveland, H., Jakobsson, K.E.: Immediate administration of tranexamic acid and reduced incidence of early rebleeding after aneurysmal subarachnoid hemorrhage: a prospective randomized study. J Neurosurg 2002; 97: 771-778.

91. Hoh, B.L., Chi, Y.Y., Dermott, M.A., Lipori, P.J., Lewis, S.B.: The effect of coiling versus clipping of ruptured and unruptured cerebral aneurysms on length of stay, hospital cost, hospital reimbursement, and surgeon reimbursement at the university of Florida. Neurosurgery 2009; 64: 614-619.

92. Hoh, B.L., Chi, Y.Y., Lawson, M.F., Mocco, J., Barker, F.G.: Length of stay and total hospital charges of clipping versus coiling for ruptured and unruptured adult cerebral aneurysms in the Nationwide Inpatient Sample database 2002 to 2006. Stroke 2010; 41: 337-342.

93. Hoh, B.L., Putman, C.M., Budzik, R.F., Carter, B.S., Ogilvy, C.S.: Combined surgical and endovascular techniques of flow alteration to treat fusiform and complex wide-necked intracranial aneurysms that are unsuitable for clipping or coil embolization. J Neurosurg 2001; 95: 24-35.

94. Huang, J. van Gelder, J.M.: The probability of sudden death from rupture of intracranial aneurysms: a meta-analysis. Neurosurgery 2002; 51: 1101-1105.

95. Hunt, M.A. Bhardwaj, A.: Caveats for triple-H therapy in the management of vasospasm after aneurysmal subarachnoid hemorrhage. Crit Care Med 2007; 35: 1985-1986.

96. Hunt, W.E. Hess, R.M.: Surgical risk as related to time of intervention in the repair of intracranial aneurysms. J Neu- 
rosurg 1968; 28: 14-20.

97. Hutchinson, P.J., Power, D.M., Tripathi, P., Kirkpatrick, P.J.: Outcome from poor grade aneurysmal subarachnoid haemorrhage--which poor grade subarachnoid haemorrhage patients benefit from aneurysm clipping? $\mathrm{Br} \mathrm{J}$ Neurosurg 2000; 14: 105-109.

98. Inagawa, T.: Ultra-early rebleeding within six hours after aneurysmal rupture. Surg Neurol 1994; 42: 130-134.

99. Inagawa, T.: Seasonal variation in the incidence of aneurysmal subarachnoid hemorrhage in hospital- and community-based studies. J Neurosurg 2002; 96: 497-509.

100. Inagawa, T. Hirano, A.: Autopsy study of unruptured incidental intracranial aneurysms. Surg Neurol 1990; 34: 361365.

101. Inagawa, T., Tokuda, Y., Ohbayashi, N., Takaya, M., Moritake, K.: Study of aneurysmal subarachnoid hemorrhage in Izumo City, Japan. Stroke 1995; 26: 761-766.

102. Ingall, T.J., Whisnant, J.P., Wiebers, D.O., O'Fallon, W.M.: Has there been a decline in subarachnoid hemorrhage mortality? Stroke 1989; 20: 718-724.

103. Ingall, T.J. Wiebers, D.O.: Natural history of subrachnoid hemorrhage., in Whisnant JP (ed): Stroke: Populations, cohorts, and clinical trials. Boston, Mass, Butterworth-Heinemann Ltd, 1993.

104. Iplikcioglu, A.C., Berkman, M.Z.: The effect of shortterm antifibrinolytic therapy on experimental vasospasm. Surg Neurol 2003; 59: 10-16.

105. Jane, J.A., Winn, H.R., Richardson, A.E.: The natural history of intracranial aneurysms: rebleeding rates during the acute and long term period and implication for surgical management. Clin Neurosurg 1977; 24: 176-184.

106. Jarus-Dziedzic, K., Juniewicz, H., Wronski, J., Zub, W.L., Kasper, E., Gowacki, M., Mierzwa, J.: The relation between cerebral blood flow velocities as measured by TCD and the incidence of delayed ischemic deficits. A prospective study after subarachnoid hemorrhage. Neurol Res 2002; 24: 582-592.

107. Johnston, S.C., Selvin, S., Gress, D.R.: The burden, trends, and demographics of mortality from subarachnoid hemorrhage. Neurology 1998; 50: 1413-1418.

108. Juvela, S.: Rebleeding from ruptured intracranial aneurysms. Surg Neurol 1989; 32: 323-326.

109. Kassell, N.F., Sasaki, T., Colohan, A.R., Nazar, G.: Cerebral vasospasm following aneurysmal subarachnoid hemorrhage. Stroke 1985; 16: 562-572.

110. Kassell, N.F., Torner, J.C.: Aneurysmal rebleeding: a preliminary report from the Cooperative Aneurysm Study. Neurosurgery 1983; 13: 479-481.

111. Kassell, N.F., Torner, J.C., Adams, H.P., Jr.: Antifibrinolytic therapy in the acute period following aneurysmal subarachnoid hemorrhage. Preliminary observations from the Cooperative Aneurysm Study. J Neurosurg 1984; 61: 225230.
112. Kassell, N.F., Torner, J.C., Haley, E.C., Jr., Jane, J.A., Adams, H.P., Kongable, G.L: The International Cooperative Study on the Timing of Aneurysm Surgery. Part 1: Overall management results. J Neurosurg 1990; 73: 18-36.

113. Kassell, N.F., Torner, J.C., Jane, J.A., Haley, E.C., Jr., Adams, H.P.: The International Cooperative Study on the Timing of Aneurysm Surgery. Part 2: Surgical results. J Neurosurg 1990; 73: 37-47.

114. Katati, M.J., Santiago-Ramajo, S., Saura, E., Jorques, A., Pérez García, M., Martín-Linares, J.M., Minguez, A., Escamilla, F., Arjona, V.: Calidad de vida en pacientes con aneurismas intracraneales: Cirugía versus tratamiento endovascular. Neurocirugía 2006; 17: 325-332

115. Kawabe, T., Tenjin, H., Hayashi, Y., Kakita, K., Kubo, S.: Midterm prevention of rebleeding by Guglielmi detachable coils in ruptured intracranial aneurysms less than $10 \mathrm{~mm}$. Clin Neurol Neurosurg 2006; 108: 163-167.

116. King, J.T., Jr.: Epidemiology of aneurysmal subarachnoid hemorrhage. Neuroimaging Clin N Am 1997; 7: 659668.

117. King, J.T., Jr., Berlin, J.A., Flamm, E.S.: Morbidity and mortality from elective surgery for asymptomatic, unruptured, intracranial aneurysms: a meta-analysis. J Neurosurg 1994; 81: 837-842.

118. Koivisto, T., Vanninen, R., Hurskainen, H., Saari, T., Hernesniemi, J., Vapalahti, M.: Outcomes of early endovascular versus surgical treatment of ruptured cerebral aneurysms. A prospective randomized study. Stroke 2000; 31 : 2369-2377.

119. Komotar, R.J., Schmidt, J.M., Starke, R.M., Claassen, J., Wartenberg, K.E., Lee, K., Badjatia, N., Connolly, E.S., Jr., Mayer, S.A.: Resuscitation and critical care of poor-grade subarachnoid hemorrhage. Neurosurgery 2009; 64: 397-410.

120. Komotar, R.J., Zacharia, B.E., Otten, M.L., Mocco, J., Lavine, S.D.: Controversies in the endovascular management of cerebral vasospasm after intracranial aneurysm rupture and future directions for therapeutic approaches. Neurosurgery 2008; 62: 897-905.

121. Kronvall, E., Undren, P., Romner, B., Saveland, H., Cronqvist, M., Nilsson, O.G.: Nimodipine in aneurysmal subarachnoid hemorrhage: a randomized study of intravenous or peroral administration. J Neurosurg 2009; 110: 58-63.

122. Kuether, T.A., Nesbit, G.M., Barnwell, S.L.: Clinical and angiographic outcomes, with treatment data, for patients with cerebral aneurysms treated with Guglielmi detachable coils: a single-center experience. Neurosurgery 1998; 43: 1016-1025.

123. Lagares, A., de Toledo, P., Fernandez-Alen, J.A., Ibanez, J., Arikan, F., Sarabia, R., Ballenilla, F., Gabarros, A., Horcajadas, A., Rodriguez-Boto, G., de la Lama, A., Maillo, A., Delgado, P., Yacer, J.L., Dominguez, J., Arrese, I.: Base de datos multicéntrica de la hemorragia subaracnoidea espontánea del Grupo de Trabajo de Patología Vascular de la Sociedad 
Española de Neurocirugía: presentación, criterios de inclusión y desarrollo de una base de datos en internet. Neurocirugía 2008; 19: 405-415.

124. Lagares, A., Fernandez-Alen, J.A., Porto, J., Campollo, J., Ramos, A., Jimenez-Roldan, L., Lobato, R.D.: Hemorragia subaracnoidea como forma de presentación de aneurisma disecante carotídeo. Neurocirugia 2007; 18: 414419.

125. Lagares, A., Gomez, P.A., Alen, J.F., Lobato, R.D., Rivas, J.J., Alday, R., Campollo, J., de la Camara, A.G.: A comparison of different grading scales for predicting outcome after subarachnoid haemorrhage. Acta Neurochir (Wien) 2005; 147: 5-16.

126. Lagares, A., Gomez, P.A., Lobato, R.D., Alen, J.F., Alday, R., Campollo, J.: Prognostic factors on hospital admission after spontaneous subarachnoid haemorrhage. Acta Neurochir (Wien) 2001; 143: 665-672.

127. Lagares, A., Gomez, P.A., Lobato, R.D., Alen, J.F., Alday, R., Campollo, J., Gonzalez, P., de la Lama, A., Palomino, J.C., Miranda, P.: Hemorragia subaracnoidea idiopática: Comparación de los diferentes patrones de sangrado y evolución a largo plazo. Neurocirugía 2002; 13: 110-119.

128. Laidlaw, J.D., Siu, K.H.: Poor-grade aneurysmal subarachnoid hemorrhage: outcome after treatment with urgent surgery. Neurosurgery 2003; 53: 1275-1280.

129. Latorre, J.G., Chou, S.H., Nogueira, R.G., Singhal, A.B., Carter, B.S., Ogilvy, C.S., Rordorf, G.A.: Effective glycemic control with aggressive hyperglycemia management is associated with improved outcome in aneurysmal subarachnoid hemorrhage. Stroke 2009; 40: 1644-1652.

130. Le Roux, P.D., Winn, H.R.: Intracranial aneurysms and subarachnoid hemorrhage management of the poor grade patient. Acta Neurochir Suppl 1999; 72: 7-26.

131. Lee, J.W., Choi, H.G., Jung, J.Y., Huh, S.K., Lee, K.C.: Surgical strategies for ruptured blister-like aneurysms arising from the internal carotid artery: a clinical analysis of 18 consecutive patients. Acta Neurochir (Wien) 2009; 151: $125-130$.

132. Lee, K. Claassen, J.: Intraventricular hemorrhage: harmful effect is not from the pressure alone. Cerebrovasc Dis 2009; 27: 411-412.

133. Lindegaard, K.F., Nornes, H., Bakke, S.J., Sorteberg, W., Nakstad, P.: Cerebral vasospasm after subarachnoid haemorrhage investigated by means of transcranial Doppler ultrasound. Acta Neurochir Suppl (Wien) 1988; 42: 81-84.

134. Linn, F.H., Rinkel, G.J., Algra, A., van Gijn, J.: Incidence of subarachnoid hemorrhage: role of region, year, and rate of computed tomography: a meta-analysis. Stroke 1996; 27: 625-629.

135. Liu, J.K., Tenner, M.S., Gottfried, O.N., Stevens, E.A., Rosenow, J.M., Madan, N., Macdonald, J.D., Kestle, J.R., Couldwell, W.T.: Efficacy of multiple intraarterial papaverine infusions for improvement in cerebral circulation time in patients with recurrent cerebral vasospasm. J Neurosurg 2004; 100: 414-421.

136. Maeda, M., Yagishita, A., Yamamoto, T., Sakuma, H., Takeda, K.: Abnormal hyperintensity within the subarachnoid space evaluated by fluid-attenuated inversion-recovery MR imaging: a spectrum of central nervous system diseases. Eur Radiol 2003; 13 Suppl 4: L192-L201.

137. Martinez-Manas,, R., Ibanez, G., Macho, J., Gaston, F., Ferrer, E.: [A study of 234 patients with subarachnoid hemorrhage of aneurysmic and cryptogenic origin]. Neurocirugia $2002 ; 13: 181-193$.

138. Mathis, J.M., DeNardo, A., Jensen, M.E., Scott, J., Dion, J.E.: Transient neurologic events associated with intraarterial papaverine infusion for subarachnoid hemorrhage-induced vasospasm. AJNR Am J Neuroradiol 1994; 15: 1671-1674.

139. Mayberg, M.R., Batjer, H.H., Dacey, R., Diringer, M., Haley, E.C., Heros, R.C., Sternau, L.L., Torner, J., Adams, H,P., Jr., Feinberg, W.: Guidelines for the management of aneurysmal subarachnoid hemorrhage. A statement for healthcare professionals from a special writing group of the Stroke Council, American Heart Association. Stroke 1994; 25: 23152328.

140. McMahon, C.J., McDermott, P., Horsfall, D., Selvarajah, J.R., King, A.T., Vail, A.: The reproducibility of transcranial Doppler middle cerebral artery velocity measurements: implications for clinical practice. Br J Neurosurg 2007; 21: 21-27.

141. Meling, T.R., Sorteberg, A., Bakke, S.J., Slettebo, H., Hernesniemi, J., Sorteberg, W.: Blood blister-like aneurysms of the internal carotid artery trunk causing subarachnoid hemorrhage: treatment and outcome. J Neurosurg 2008; 108: 662-671.

142. Meyers, P.M., Schumacher, H.C., Higashida, R.T., Barnwell, S.L., Creager, M.A., Gupta, R., McDougall, C.G., Pandey, D.K., Sacks, D., Wechsler, L.R.: Indications for the performance of intracranial endovascular neurointerventional procedures: a scientific statement from the American Heart Association Council on Cardiovascular Radiology and Intervention, Stroke Council, Council on Cardiovascular Surgery and Anesthesia, Interdisciplinary Council on Peripheral Vascular Disease, and Interdisciplinary Council on Quality of Care and Outcomes Research. Circulation 2009; 119: 22352249.

143. Milhorat, T.H.: Timing of aneurysm surgery in subarachnoid hemorrhage: a systematic review of the literature. Neurosurgery 2002; 51: 525.

144. Miranda, P., Lagares, A., Alen, J., Perez-Nunez, A., Arrese, I., Lobato, R.D.: Early transcranial Doppler after subarachnoid hemorrhage: clinical and radiological correlations. Surg Neurol 2006; 65: 247-252.

145. Miyaoka, M., Sato, K., Ishii, S.: A clinical study of the relationship of timing to outcome of surgery for ruptured 
cerebral aneurysms. A retrospective analysis of 1622 cases. J Neurosurg 1993; 79: 373-378.

146. Molyneux, A.J., Kerr, R.S., Yu, L.M., Clarke, M., Sneade, M., Yarnold, J.A., Sandercock, P.: International subarachnoid aneurysm trial (ISAT) of neurosurgical clipping versus endovascular coiling in 2143 patients with ruptured intracranial aneurysms: a randomised comparison of effects on survival, dependency, seizures, rebleeding, subgroups, and aneurysm occlusion. Lancet 2005; 366: 809-817.

147. Morales, F., Maillo, A., Hernández, J., Pastor, A., Caballero, M., Gómez Moreta, J., Díaz, P. Santamarta, D.: Evaluación del tratamiento microquirúrgico de 121 aneurismas intracraneales. Neurocirugía 2003; 14: 5-15.

148. Morgan, M.K., Jonker, B., Finfer, S., Harrington, T., Dorsch, N.W.: Aggressive management of aneurysmal subarachnoid haemorrhage based on a papaverine angioplasty protocol. J Clin Neurosci 2000; 7: 305-308.

149. Morgenstern, L.B., Luna-Gonzales, H., Huber, J.C., Jr., Wong, S.S., Uthman, M.O., Gurian, J.H., Castillo, P.R., Shaw, S.G., Frankowski, R.F., Grotta, J.C.: Worst headache and subarachnoid hemorrhage: prospective, modern computed tomography and spinal fluid analysis. Ann Emerg Med 1998; 32: 297-304.

150. Munoz-Sanchez, M.A., Cayuela-Dominguez, A., Murillo-Cabezas, F., Navarrete-Navarro, P., Munoz-Lopez, A., Guerrero-Lopez, F., Garcia-Alfaro, C., Jimenez-Moragas, J.M., Martinez-Escobar, S., de la Torre-Prados, M.V.: [Improving the outcomes in spontaneous subarachnoid haemorrhage: the EHSA project]. Rev Neurol 2009; 49: 399-404.

151. Murayama, Y., Nien, Y.L., Duckwiler, G., Gobin, Y.P., Jahan, R., Frazee, J., Martin, N., Vinuela, F.: Guglielmi detachable coil embolization of cerebral aneurysms: 11 years' experience. J Neurosurg 2003; 98: 959-966.

152. Naff, N.J., Hanley, D.F., Keyl, P.M., Tuhrim, S., Kraut, M., Bederson, J., Bullock, R., Mayer, S.A., Schmutzhard, E.: Intraventricular thrombolysis speeds blood clot resolution: results of a pilot, prospective, randomized, doubleblind, controlled trial. Neurosurgery 2004; 54: 577-583.

153. Natarajan, S.K., Sekhar, L.N., Ghodke, B., Britz, G.W., Bhagawati, D., Temkin, N.: Outcomes of ruptured intracranial aneurysms treated by microsurgical clipping and endovascular coiling in a high-volume center. AJNR Am J Neuroradiol 2008; 29: 753-759.

154. Naval, N.S., Thomas, C.E., Urrutia, V.C.: Relative changes in flow velocities in vasospasm after subarachnoid hemorrhage: a transcranial Doppler study. Neurocrit Care 2005; 2:1 33-140.

155. Nibbelink, D.W., Torner, J.C., Henderson, W.G.: Intracranial aneurysms and subarachnoid hemorrhage. A cooperative study. Antifibrinolytic therapy in recent onset subarachnoid hemorrhage. Stroke 1975; 6: 622-629.

156. Niesen, W.D., Rosenkranz, M., Schummer, W., Weiller, C., Sliwka, U.: Cerebral venous flow velocity predicts poor outcome in subarachnoid hemorrhage. Stroke 2004; 35: 1873-1878.

157. Nieuwkamp, D.J., de Gans, K., Algra, A., Albrecht, K.W., Boomstra, S., Brouwers, P.J., Groen, R.J., Metzemaekers, J.D., Nijssen, P.C., Roos, Y.B., Tulleken, C.A., Vandertop, W.P., van Gijn, J., Vos, P.E., Rinkel, G.J.: Timing of aneurysm surgery in subarachnoid haemorrhage--an observational study in The Netherlands. Acta Neurochir (Wien) 2005; 147: 815-821.

158. Nieuwkamp, D.J., de Gans, K., Rinkel, G.J., Algra, A.: Treatment and outcome of severe intraventricular extension in patients with subarachnoid or intracerebral hemorrhage: a systematic review of the literature. J Neurol 2000; 247: $117-121$.

159. Nieuwkamp, D.J., Setz, L.E., Algra, A., Linn, F.H., de Rooij, N.K., Rinkel, G.J.: Changes in case fatality of aneurysmal subarachnoid haemorrhage over time, according to age, sex, and region: a meta-analysis. Lancet Neurol 2009; 8: 635642 .

160. Nishioka, H., Torner, J.C., Graf, C.J., Kassell, N.F., Sahs, A.L., Goettler, L.C.: Cooperative study of intracranial aneurysms and subarachnoid hemorrhage: a long-term prognostic study. II. Ruptured intracranial aneurysms managed conservatively. Arch Neurol 1984; 41: 1142-1146.

161. Nishioka, H., Torner, J.C., Graf, C.J., Kassell, N.F., Sahs, A.L., Goettler, L.C.: Cooperative study of intracranial aneurysms and subarachnoid hemorrhage: a long-term prognostic study. III. Subarachnoid hemorrhage of undetermined etiology. Arch Neurol 1984; 41: 1147-1151.

162. Nornes, H.: Cerebral arterial flow dynamics during aneurysm haemorrhage. Acta Neurochir (Wien) 1978; 41: 3948.

163. Ogilvy, C.S., Carter, B.S.: A proposed comprehensive grading system to predict outcome for surgical management of intracranial aneurysms. Neurosurgery 1998; 42: 959-968.

164. Ogilvy, C.S., Carter, B.S., Kaplan, S., Rich, C., Crowell, R.M.: Temporary vessel occlusion for aneurysm surgery: risk factors for stroke in patients protected by induced hypothermia and hypertension and intravenous mannitol administration. J Neurosurg 1996; 84: 785-791.

165. Ohman, J., Heiskanen, O.: Timing of operation for ruptured supratentorial aneurysms: a prospective randomized study. J Neurosurg 1989; 70: 55-60.

166. Ohman, J., Servo, A., Heiskanen, O.: Long-term effects of nimodipine on cerebral infarcts and outcome after aneurysmal subarachnoid hemorrhage and surgery. J Neurosurg 1991; 74: 8-13.

167. Origitano, T.C., Wascher, T.M., Reichman, O.H., Anderson, D.E.: Sustained increased cerebral blood flow with prophylactic hypertensive hypervolemic hemodilution ("triple-H" therapy) after subarachnoid hemorrhage. Neurosurgery $1990 ; 27: 729-739$.

168. Orozco-Giménez, C., Katati, M.J., Vilar, R., Meers- 
mans, M., Pérez García, M., Martín, J.M., Alcazar, P., Guerrero, F., Escamilla, F., Minguez, A., Olivares, G., Saura, E., Jorques, A., Arjona, V.: Alteraciones neuropsicológicas en pacientes con aneurismas cerebrales: Tratamiento quirúrgico versus tratamiento endovascular. Neurocirugía 2006; 17: 3445

169. Pakarinen, S.: Incidence, aetiology, and prognosis of primary subarachnoid haemorrhage. A study based on 589 cases diagnosed in a defined urban population during a defined period. Acta Neurol Scand 1967; 43: Suppl-28.

170. Phillips, L.H., Whisnant, J.P., O'Fallon, W.M., Sundt, T.M., Jr.: The unchanging pattern of subarachnoid hemorrhage in a community. Neurology 1980; 30: 1034-1040.

171. Pickard, J.D., Murray, G.D., Illingworth, R., Shaw, M.D., Teasdale, G.M., Foy, P.M., Humphrey, P.R., Lang, D.A., Nelson, R., Richards, P.: Oral nimodipine and cerebral ischaemia following subarachnoid haemorrhage. Br J Clin Pract 1990; 44: 66-67.

172. Qureshi, A.I., Suri, M.F., Yahia, A.M., Suarez, J.I., Guterman, L.R., Hopkins, L.N., Tamargo, R.J.: Risk factors for subarachnoid hemorrhage. Neurosurgery 2001; 49: 607612.

173. Raaymakers, T.W., Buys, P.C., Verbeeten, B., Jr., Ramos, L.M., Witkamp, T.D., Hulsmans, F.J., Mali, W.P., Algra, A., Bonsel, G.J., Bossuyt, P.M., Vonk, C.M., Buskens, E., Limburg, M., van Gijn, J., Gorissen, A., Greebe, P., Albrecht, K.W., Tulleken, C.A., Rinkel, G.J.: MR angiography as a screening tool for intracranial aneurysms: feasibility, test characteristics, and interobserver agreement. AJR Am J Roentgenol 1999; 173: 1469-1475.

174. Raaymakers, T.W., Rinkel, G.J., Ramos, L.M.: Initial and follow-up screening for aneurysms in families with familial subarachnoid hemorrhage. Neurology 1998; 51: 11251130 .

175. Rinkel, G.J.: Natural history, epidemiology and screening of unruptured intracranial aneurysms. Rev Neurol (Paris) 2008; 164: 781-786.

176. Rinkel, G.J., Wijdicks, E.F., Hasan, D., Kienstra, G.E., Franke, C.L., Hageman, L.M., Vermeulen, M., van Gijn, J.: Outcome in patients with subarachnoid haemorrhage and negative angiography according to pattern of haemorrhage on computed tomography. Lancet 1991; 338: 964-968.

177. Roda, J.M., Gonzalez-Llanos, F., Pascual, J.M.: [The role of the extra-intracranial anastomosis and interventionist endovascular therapy in the treatment of complex cerebral aneurysms]. Neurocirugia 2002; 13: 365-370.

178. Roda, J.M., Conesa, G., Lobato, R.D., Garcia Allut, A., Gomez, P.A., Gonzalez Darder, J.M., Lagares, A.: Hemorragia subaranoidea aneurismática. Introducción a alguno de los aspectos más importantes de esta enfermedad. Neurocirugía 2000; 11: 156-168.

179. Romner, B., Reinstrup, P.: Triple H therapy after aneurysmal subarachnoid hemorrhage. A review. Acta Neuro- chir Suppl 2001; 77: 237-241.

180. Roos, Y.B., Beenen, L.F., Groen, R.J., Albrecht, K.W., Vermeulen, M.: Timing of surgery in patients with aneurysmal subarachnoid haemorrhage: rebleeding is still the major cause of poor outcome in neurosurgical units that aim at early surgery. J Neurol Neurosurg Psychiatry 1997; 63: 490-493.

181. Rordorf, G., Ogilvy, C.S., Gress, D.R., Crowell, R.M., Choi, I.S.: Patients in poor neurological condition after subarachnoid hemorrhage: early management and long-term outcome. Acta Neurochir (Wien) 1997; 139: 1143-1151.

182. Ross, N., Hutchinson, P.J., Seeley, H., Kirkpatrick, P.J.: Timing of surgery for supratentorial aneurysmal subarachnoid haemorrhage: report of a prospective study. J Neurol Neurosurg Psychiatry 2002; 72: 480-484.

183. Rubio, E., Castaño, C., Vilalta, J., García Díez, J., Sahuquillo, J.: Clipaje transitorio de la arteria aferente al saco aneurismático en la cirugía de aneurismas intracraneales. Neurocirugía 1990; 1: 375-383

184. Sacco, S., Totaro, R., Toni, D., Marini, C., Cerone, D., Carolei, A.: Incidence, case-fatalities and 10-year survival of subarachnoid hemorrhage in a population-based registry. Eur Neurol 2009; 62: 155-160.

185. Sames, T.A., Storrow, A.B., Finkelstein, J.A., Magoon, M.R.: Sensitivity of new-generation computed tomography in subarachnoid hemorrhage. Acad Emerg Med 1996; 3: 16-20.

186. Sánchez Casado, M., Sánchez Ledesma, M.J., Gonçalves, J., Abad, M., García March, G., Broseta, J.: Efecto de la hipotermia combinada con magnesio y tirilazad en un modelo experimental de isquemia cerebral difusa. Med Intensiva 2007; 31: 113-119

187. Sano, K., Saito, I.: Timing and indication of surgery for ruptured intracranial aneurysms with regard to cerebral vasospasm. Acta Neurochir (Wien) 1978; 41: 49-60.

188. Sarabia, R., Lagares, A., Alen, J.F., Arikan, F., Vilalta, J., Ibanez, J., Maillo, A.: Idiopathic subarachnoid hemorrhage: a multicentre series of 220 patients. Neurocirugia 2010; 21 : 441-451.

189. Saveland, H., Hillman, J., Brandt, L., Edner, G., Jakobsson, K.E., Algers, G.: Overall outcome in aneurysmal subarachnoid hemorrhage. A prospective study from neurosurgical units in Sweden during a 1-year period. J Neurosurg 1992; 76: 729-734.

190. Schievink, W.I., Limburg, M., Oorthuys, J.W., Fleury, P., Pope, F.M.: Cerebrovascular disease in Ehlers-Danlos syndrome type IV. Stroke 1990; 21: 626-632.

191. Schievink, W.I., Wijdicks, E.F., Piepgras, D.G., Chu, C.P., O'Fallon, W.M., Whisnant, J.P.: The poor prognosis of ruptured intracranial aneurysms of the posterior circulation. J Neurosurg 1995; 82: 791-795.

192. Schmidt, U., Bittner, E., Pivi, S., Marota, J.J.: Hemodynamic management and outcome of patients treated for cerebral vasospasm with intraarterial nicardipine and/or mil- 
rinone. Anesth Analg 2010; 110: 895-902.

193. Seifert, V., Stolke, D., Trost, H.A.: Timing of aneurysm surgery. Comparison of results of early and delayed surgical intervention. Eur Arch Psychiatry Neurol Sci 1988; 237 : 291-297.

194. Seifert V, Trost HA, and Stolke D Management morbidity and mortality in grade IV and V patients with aneurysmal subarachnoid haemorrhage. Acta Neurochir (Wien) 1990; 103: 5-10.

195. Sengupta, R.P., McAllister, V.L: Subarachnoid hemorrhage. 1986.

196. Shah, D., Husain, A.M.: Utility of levetiracetam in patients with subarachnoid hemorrhage. Seizure 2009; 18: 676-679.

197. Sidman, R., Connolly, E., Lemke, T.: Subarachnoid hemorrhage diagnosis: lumbar puncture is still needed when the computed tomography scan is normal. Acad Emerg Med 1996; 3: 827-831.

198. Sloffer, C.A., Augspurger, L., Wagenbach, A., Lanzino, G.: Antimicrobial-impregnated external ventricular catheters: does the very low infection rate observed in clinical trials apply to daily clinical practice? Neurosurgery 2005; 56 : 1041-1044.

199. Sluzewski, M., Menovsky, T., van Rooij, W.J., Wijnalda, D.: Coiling of very large or giant cerebral aneurysms: long-term clinical and serial angiographic results. AJNR Am J Neuroradiol 2003; 24: 257-262.

200. Sluzewski, M., van Rooij, W.J., Beute, G.N., Nijssen, P.C.: Late rebleeding of ruptured intracranial aneurysms treated with detachable coils. AJNR Am J Neuroradiol 2005; 26 : 2542-2549.

201. Sorteberg, A., Bakke, S.J., Boysen, M., Sorteberg, W.: Angiographic balloon test occlusion and therapeutic sacrifice of major arteries to the brain. Neurosurgery 2008; 63: 651660.

202. Starke, R.M., Kim, G.H., Fernandez, A., Komotar, R.J., Hickman, Z.L., Otten, M.L., Ducruet, A.F., Kellner, C.P., Hahn, D.K., Chwajol, M., Mayer, S.A., Connolly, E.S., Jr.: Impact of a protocol for acute antifibrinolytic therapy on aneurysm rebleeding after subarachnoid hemorrhage. Stroke 2008; 39: 2617-2621.

203. Sundt, T.M., Jr.: Surgical Techniques for saccular and gian intracranial aneurysms. Baltimore, Maryland, Williams and Wilkins, 1990.

204. Szaflarski, J.P., Sangha, K.S., Lindsell, C.J., Shutter, L.A.: Prospective, randomized, single-blinded comparative trial of intravenous levetiracetam versus phenytoin for seizure prophylaxis. Neurocrit Care 2010; 12: 165-172.

205. Taylor, C.L., Yuan, Z., Selman, W.R., Ratcheson, R.A., Rimm, A.A.: Cerebral arterial aneurysm formation and rupture in 20,767 elderly patients: hypertension and other risk factors. J Neurosurg 1995; 83: 812-819.

206. Teunissen, L.L., Rinkel, G.J., Algra, A., van Gijn,
J.: Risk factors for subarachnoid hemorrhage: a systematic review. Stroke 1996; 27: 544-549.

207. Tiel Groenestege, A.T., Rinkel, G.J., van der Bom, J.G., Algra, A., Klijn, C.J.: The risk of aneurysmal subarachnoid hemorrhage during pregnancy, delivery, and the puerperium in the Utrecht population: case-crossover study and standardized incidence ratio estimation. Stroke 2009; 40: 1148-1151.

208. Torres, A., Plans, G., Martino, J., Godino, O., Garcia, I., Gracia, B., Acebes, J.J.: Fibrinolytic therapy in spontaneous intraventricular haemorrhage: efficacy and safety of the treatment. Br J Neurosurg 2008; 22: 269-274.

209. Treggiari, M.M., Deem, S.: Which H is the most important in triple-H therapy for cerebral vasospasm? Curr Opin Crit Care 2009; 15: 83-86.

210. Ujiie, H., Sato, K., Onda, H., Oikawa, A., Kagawa, M., Takakura, K., Kobayashi, N.: Clinical analysis of incidentally discovered unruptured aneurysms. Stroke 1993; 24: 1850-1856.

211. van der W.N., Rinkel, G.J., Hasan, D., van Gijn, J.: Detection of subarachnoid haemorrhage on early CT: is lumbar puncture still needed after a negative scan? J Neurol Neurosurg Psychiatry 1995; 58: 357-359.

212. van Gijn, J., Kerr, R.S., Rinkel, G.J.: Subarachnoid haemorrhage. Lancet 2007; 369: 306-318.

213. van dS, I., Velthuis, B.K., Wermer, M.J., Majoie, C., Witkamp, T., de Kort, G., Freling, N.J., Rinkel, G.J.: New detected aneurysms on follow-up screening in patients with previously clipped intracranial aneurysms: comparison with DSA or CTA at the time of SAH. Stroke 2005; 36: 17531758 .

214. Varelas, P.N., Rickert, K.L., Cusick, J., Hacein-Bey, L., Sinson, G., Torbey, M., Spanaki, M., Gennarelli, T.A.: Intraventricular hemorrhage after aneurysmal subarachnoid hemorrhage: pilot study of treatment with intraventricular tissue plasminogen activator. Neurosurgery 2005; 56: 205213.

215. Vega-Basulto, S.D., Lafontaine, E., Gutierrez, F., Roura, J., Pardo, G.: Hemorragia intracraneal por aneurismas y malformaciones arteriovenosas durante el embarazo y el puerperio. Neurocirugía 2008; 19: 25-34.

216. Vega-Basulto, S.D., Silva, S., Peñones, R.: Tratamiento quirúrgico de los aneurismas intracraneales múltiples. Neurocirugía 2003; 14: 385-391.

217. Velthuis, B.K., van Leeuwen, M.S., Witkamp, T.D., Ramos, L.M., Berkelbach van der Sprenkel, J.W., Rinkel, G.J.: Computerized tomography angiography in patients with subarachnoid hemorrhage: from aneurysm detection to treatment without conventional angiography. J Neurosurg 1999; 91: 761-767.

218. Vinuela, F., Duckwiler, G., Mawad, M.: Guglielmi detachable coil embolization of acute intracranial aneurysm: perioperative anatomical and clinical outcome in 403 patients. 
J Neurosurg 1997; 86: 475-482.

219. Wermer, M.J., Buskens, E., van dS, I., Bossuyt, P.M., Rinkel, G.J.: Yield of screening for new aneurysms after treatment for subarachnoid hemorrhage. Neurology 2004; 62: 369375.

220. Wermer, M.J., Rinkel, G.J., van Gijn, J.: Repeated screening for intracranial aneurysms in familial subarachnoid hemorrhage. Stroke 2003; 34: 2788-2791.

221. Wermer, M.J., van dS, I., Algra, A., Rinkel, G.J.: Risk of rupture of unruptured intracranial aneurysms in relation to patient and aneurysm characteristics: an updated meta-analysis. Stroke 2007; 38: 1404-1410.

222. Wermer, M.J., van dS, I., Velthuis, B.K., Algra, A., Buskens, E., Rinkel, G.J.: Follow-up screening after subarachnoid haemorrhage: frequency and determinants of new aneurysms and enlargement of existing aneurysms. Brain 2005; 128: 2421-2429.

223. Whisnant, J.P., Sacco, S.E., O'Fallon, W.M., Fode, N.C., Sundt, T.M., Jr.: Referral bias in aneurysmal subarachnoid hemorrhage. J Neurosurg 1993; 78: 726-732.

224. Whitfield, P.C., Kirkpatrick, P.J.: Timing of surgery for aneurysmal subarachnoid haemorrhage. Cochrane Database Syst Rev 2001; CD001697.

225. Winn, H.R., Richardson, A.E., Jane, J.A.: The long-term prognosis in untreated cerebral aneurysms: I. The incidence of late hemorrhage in cerebral aneurysm: a 10-year evaluation of 364 patients. Ann Neurol 1977; 1: 358-370.

226. Winn, H.R., Richardson, A.E., O’Brien, W., Jane, J.A.: The long-term prognosis in untreated cerebral aneu- rysms: II. Late morbidity and mortality. Ann Neurol 1978; 4: 418-426.

227. Yasargil, M.G.: Microneurosurgery. Vols 1\&2. Stuttgart, New York, Thieme, 1984.

228. Yoshimoto, Y., Wakai, S., Satoh, A., Tejima, T., Hamano, M.: A prospective study on the effects of early surgery on vasospasm after subarachnoid hemorrhage. Surg Neurol 1999; 51: 392-397.

229. Zabramski, J.M., Whiting, D., Darouiche, R.O., Horner, T.G., Olson, J., Robertson, C., Hamilton, A.J.: Efficacy of antimicrobial-impregnated external ventricular drain catheters: a prospective, randomized, controlled trial. J Neurosurg 2003; 98: 725-730.

230. Zubkov, A.Y., Wijdicks, E.F.: Antiepileptic drugs in aneurysmal subarachnoid hemorrhage. Rev Neurol Dis 2008; 5: 178-181.

231. Zubkov, Y.N., Nikiforov, B.M., Shustin, V.A.: Balloon catheter technique for dilatation of constricted cerebral arteries after aneurysmal SAH. Acta Neurochir (Wien) 1984; 70: 65-79.

Lagares, A.; Gómez, P.A.; Alén, J.F.; Arikan, F.; Sarabia, R.; Horcajadas, A.; Ibañez, J.; Gabarros, A.; Morera, J.; de la Lama, A.; Ley, L.; Gonçalves, J.; Maillo, A.; Domínguez, J.; Llacer, J.L.; Arrese, I.; Santamarta, D.; Delgado, P.; Rodríguez Boto, G.; Vilalta, J.: Hemorragia subaracnoidea aneurismática: guía de tratamiento del Grupo de Patología Vascular de la Sociedad Española de Neurocirugía. Neurocirugía 2011; 22: 93-115. 